\title{
Anästhesie zur Karotischirurgie
}

\section{Gibt es einen Goldstandard?}

\begin{abstract}
Aufgrund der ätiologiebedingten, meist vorbestehenden signifikanten neurologischen und kardialen Komorbidität sowie der Komplexität der physiologischen Reaktionen auf die Thrombendarteriektomie (TEA) der A. carotis selbst stellt die perioperative Betreuung der Patienten mit symptomatischen Karotisstenosen höchste Anforderungen an Anästhesist und Operateur. Die TEA wird sowohl unter Regionalanästhesie als auch Allgemeinanästhesie durchgeführt. Bislang dominieren in Deutschland eindeutig die Allgemeinanästhesieverfahren, der Anteil an Regionalanästhesietechniken nimmt aber kontinuierlich zu. Unabhängig vom gewählten Anästhesieverfahren muss sichergestellt werden, dass der Anästhesist Vor- und Nachteile des jeweiligen Verfahrens sowie dessen Risiken kennt und dieses auf den individuellen Patienten ausrichtet.
\end{abstract}

\section{Grundlagen}

Mit einer Prävalenz von ca. 200.000 zerebralen Insulten/Jahr stellt der Schlaganfall eine der wichtigsten Ursachen für Morbidität und Mortalität in Deutschland dar [25, 88]. Die Inzidenz ist dabei altersabhängig und beträgt bis zum 64. Lebensjahr etwa 300/100.000 Einwohner und Jahr, während diese bei $\mathrm{Pa}$ tienten jenseits des 85 . Lebensjahres auf mehr als 2400/100.000 Einwohner und Jahr ansteigt [26]. Mit ca. $80 \%$ ist die zerebrale Ischämie die wichtigste Ursache des Schlaganfalls. Lediglich in $20 \%$ der Fälle kann eine intrazerebrale Blutung als Auslöser identifiziert werden. Etwa $20 \%$ der Schlaganfälle, die auf dem Boden einer zerebralen Ischämie stattfinden, sind direkte Folge stenotischer Veränderungen der A. carotis interna (ACI; $[8,76])$. Das Schlaganfallrisiko ist dabei vom Grad der Stenose abhängig. Das Risiko eines Insults beträgt für Stenosen $>50 \% 1-2 \% /$ Jahr. Für hochgradige ACI-Stenosierungen mit einem Grad $>80 \%$ kann dieses Risiko bis auf etwa $3 \% / J a h r$ ansteigen $[1,27]$. In mehr als 90\% der Fälle entstehen die Stenosen aufgrund einer Atherosklerose, deren hauptsächliche Risikofaktoren die arterielle Hypertonie, ein höheres Lebensalter, die Hyperlipoproteinämie und der Nikotinabusus sind. Seltene Ursachen für eine ACI-Stenosierung stellen die ACI-Dissektionen, die fibromuskuläre Dysplasie und Rezidivstenosen nach Thrombendarterektomie (TEA; [5, 64]) dar. Bevorzugte Lokalisationen der Stenosen sind hierbei der Abgang der ACI und der Karotisbulbus. Die Diagnostik zur Bestimmung des Stenosegrades stützt sich neben Anamnese und klinischer Untersuchung v. a. auf die noninvasiven Verfahren der Doppler- und Duplexsonograpie. Diese noninvasive Diagnostik ist jedoch stark von der Erfahrung des Untersuchers abhängig. Bei nichteindeutigen Befunden steht mit der selektiven Karotisangiographie ein invasives Verfahren mit hoher Sensitivität und Spezifität zur Quantifizierung einer ACIStenose zur Verfügung.

Die differenzierte klinische Einteilung erfolgt in symptomatische und asymptomatische Karotisstenosen. Im deutschsprachigen Raum ist die Klassifikation in
4 Stadien nach Vollmar üblich (• Tab. 1; [87]).

Trotz neuer invasiver $[41,73,91]$ und medikamentöser $[18,84]$ Therapieoptionen stellt die TEA der ACI zurzeit die evidenzbasierte Maßnahme in der kurativen Behandlung symptomatischer Karotisstenosen dar, wohingegen die Indikation zur operativen Therapie asymptomatischer Stenosen streng gestellt werden muss $[28,63]$. So wurde der Stellenwert der TEA bei asymptomatischen Stenosen im Vergleich zur alleinigen Thrombozytenaggregationshemmertherapie in verschiedenen Studien untersucht. Der Präventiveffekt der TEA bezüglich des Endpunktes ,ipsilateraler Schlaganfall“ wird hierbei durch die „absolute Risikoreduktion" (ARR) ausgedrückt. Während methodische Mängel die Aussagekraft der CASANOVA-Studie sowie der ACAS-Studie reduzieren, erbrachte die nordamerikanische VA-Studie Nr. 167 hinsichtlich des Zielkriteriums ,,ipsilateraler Schlaganfall“ keine statistisch signifikanten Unterschiede $[1,15,17]$. Im Gegensatz zu diesen Untersuchungen zeigte die europäische ACST-Studie eine signifikate ARR von 5,4\% für den Einsatz der TEA [36]. Hinsichtlich der operativen Therapie symptomatischer Stenosen ist die Studienlage wesentlich eindeutiger und die Frage nach dem Benefit leichter zu beantworten. So untersuchten der European Carotid Trail (ECST) und der North American Symptomatic Carotid Endarterecto-

Thomas Rössel und Rainer J. Litz haben zu gleichen Teilen zur Abfassung des Manuskriptes beigetragen. 


\begin{tabular}{|c|c|}
\hline Stadium & Klinische Symptome \\
\hline \multirow[t]{3}{*}{ I } & Asympomatische Stenose \\
\hline & Ohne hochgradige kontralaterale Stenose bzw. Verschluss \\
\hline & Mit hochgradiger kontralateraler Stenose bzw. Verschluss \\
\hline \multirow{3}{*}{$A$} & Reversible zerebrale Ischämie $<6$ Monate \\
\hline & Amaurosis fugax \\
\hline & TIA (Symptome $<24 \mathrm{~h}$ ) \\
\hline III & Indikationen zur Notfall-Karotis-TEA \\
\hline A & Krescendo-TIA \\
\hline B & Akuter/progredienter Schlaganfall \\
\hline IV & Ipsilateraler Schlaganfall $<6$ Monate \\
\hline \multicolumn{2}{|c|}{ TIA transitorisch ischämische Attacke, TEA Thrombendarterektomie. } \\
\hline
\end{tabular}

Tab. 2 Vergleich des Schlaganfallrisikos in Abhängigkeit vom Grad der A.-carotis-interna-Stenose und der Wahl des Therapieverfahrens

\begin{tabular}{|c|c|c|c|c|c|}
\hline \multirow{2}{*}{$\begin{array}{l}\text { Stenosegrad } \\
\text { [\%] }\end{array}$} & \multirow{2}{*}{$\begin{array}{l}\text { Operationsrisiko } \\
\text { [\%] }\end{array}$} & \multicolumn{2}{|c|}{ Schlaganfallrisiko [\%] } & \multirow{2}{*}{$\begin{array}{l}\text { Absolute Risiko- } \\
\text { reduktion [\%] }\end{array}$} & \multirow[t]{2}{*}{ p } \\
\hline & & Operativ & Konservativ & & \\
\hline$<30$ & 6,7 & 12,0 & 10,0 & $-2,2$ & 0,05 \\
\hline $30-49$ & 8,4 & 15,0 & 18,2 & 3,2 & 0,6 \\
\hline $50-69$ & 8,4 & 14,0 & 18,6 & 4,6 & 0,04 \\
\hline 70-99 & 6,2 & 10,0 & 26,0 & 15,9 & $<0,001$ \\
\hline \multicolumn{6}{|c|}{$\begin{array}{l}\text { Zusammenfassung der Ergebnisse aus NASCET, ECST und VAT [28, 51, 63]. } \\
\text { Operationsrisiko: Risiko für, „Stroke“ oder Tod bis } 24 \text { h postoperativ. } \\
\text { Langzeitrisiko in Studiengruppen für Stroke oder Tod studienabhängig } 1 \text { Jahre [51], } 2 \text { Jahre [63] oder } 6 \text { Jahre [28] } \\
\text { (Schlaganfallrisiko). } \\
\text { p Signifikanzniveau zwischen den Studiengruppen. }\end{array}$} \\
\hline
\end{tabular}

\begin{tabular}{|c|c|c|c|c|c|c|}
\hline \multirow[t]{2}{*}{ Jahr } & \multirow[t]{2}{*}{ Patientenanzahl } & \multicolumn{5}{|c|}{ ASA-Klassifikation } \\
\hline & & I & II & III & IV & v \\
\hline 2002 & 15694 & 3,25 & 35,91 & 56,91 & 3,89 & 0,04 \\
\hline 2003 & 19355 & 3,01 & 32,73 & 60,45 & 3,70 & 0,11 \\
\hline 2004 & 23502 & 2,13 & 31,14 & 63,45 & 3,20 & 0,08 \\
\hline 2005 & 25629 & 1,94 & 29,89 & 64,86 & 3,27 & 0,05 \\
\hline 2006 & 25570 & 2,27 & 29,34 & 65,31 & 3,03 & 0,06 \\
\hline
\end{tabular}

Tab. 4 Anteil der Operationen unter Verwendung eines technischen Neuromonitoringverfahrens und prozentuale Verteilung der angewendeten Verfahren in Deutschland. (Bundesgeschäftsstelle für Qualitätssicherung [12])

\begin{tabular}{|llllll}
\multicolumn{5}{c}{ Erfassungsjahr } \\
\cline { 2 - 6 } & $\mathbf{2 0 0 2}$ & $\mathbf{2 0 0 3}$ & $\mathbf{2 0 0 4}$ & $\mathbf{2 0 0 5}$ & $\mathbf{2 0 0 6}$ \\
\hline Operationen & 15.694 & 19.355 & 23.502 & 25.629 & 25.570 \\
\hline Neuromonitoring & 7663 & 9642 & 12.282 & 13.393 & 13.166 \\
\hline SEP [\%] & 64,95 & 67,05 & 65,14 & 61,55 & 58,90 \\
\hline Oxymetrie [\%] & 17,55 & 18,09 & 19,30 & 18,73 & 17,34 \\
\hline EEG [\%] & 12,55 & 13,20 & 10,19 & 11,37 & 11,99 \\
\hline Sonstiges [\%] & 18,77 & 15,89 & 20,96 & 24,52 & 26,69 \\
\hline EEG Elektroenzephalogramm, SEP somatosensorisch evozierte Potenziale. & & \\
\hline
\end{tabular}

my Trail (NASCET) das Outcome der Patienten mit symptomatischen ACI-Stenosen unter operativer Therapie plus „best medical treatment" im Vergleich zu einer ment" eine hochsignifikante Risikoreduktion beim Vorliegen einer 70- bis 90\%igen Stenose bezüglich der Endpunkte „ipsilateraler Schlaganfall“ bzw. „ipsilateraler invalidisierender oder tödlicher Schlaganfall“ (• Tab. 2). Aufgrund einer Rate ipsilateraler Schlaganfälle von $26 \%$ in der konservativ behandelten Gruppe vs. $9 \%$ in der operativen Gruppe wurde in der NASCET-Studie die Untersuchung für 70- bis 99\%ige Stenosen aus ethischen Gründen vorzeitig abgebrochen [28, 63].

\section{Operationstechnik und Shunt}

Das Prinzip der TEA besteht in der Desobliteration der atherosklerotischen Plaque. Hierzu existieren zwei Operationstechniken (• Abb. 1): die konventionelle TEA mit oder ohne Patchplastik und die Eversions-TEA.

Nach Angaben der Gesellschaft für Gefäßchirurgie beträgt der Anteil der konventionellen TEA in Deutschland 63\%, der Anteil der Eversions-TEA 37\% [12]. Die Wahl der Operationstechnik scheint keinen signifikanten Einfluss auf das Operationsrisiko oder das postoperative Ergebnis zu haben [13]. Beiden Operationstechniken gemeinsam ist die einseitig temporäre Unterbrechung des Blutflusses. Dieses „clamping“ wird von ca. $90 \%$ der Patienten ohne ernsthafte Beeinträchtigung der neurologischen Funktion toleriert. Der Grund hierfür liegt in der redundanten Blutversorgung über den an der Hirnbasis gelegenen Circulus arteriosus cerebri (Circulus Willisii) und zusätzlicher Kollateralkreisläufe über den Kortex. Diese Umgehungskreisläufe sind jedoch individuell unterschiedlich stark ausgebildet und von den meist im übrigen arteriellen Kreislauf ebenfalls vorhandenen atherosklerotischen Veränderungen gleichfalls betroffen [35]. Es ist kaum möglich bereits im Vorfeld der Operation zuverlässige Aussagen über die Effektivität dieser Kollateralversorgung zu treffen, da hierfür bislang keine verlässliche klinische Untersuchungsmethode zur Verfügung steht $[4,42,43]$. Seit den ersten Erfahrungen in der Karotischirurgie sehen die meisten Autoren in der Einlage eines intraluminalen Shunts die effektivste Methode zur Sicherstellung eines ausreichenden Blutflusses in den 
abhängigen Hirnarealen. Allerdings wird die Frage, ob eine Shunteinlage routinemäßig oder selektiv erfolgen sollte, nicht eindeutig beantwortet [77]. Kritiker der routinemäßigen Shuntanwendung postulieren eine erhöhte Rate an Intimaschäden, Embolien und Nachblutungen. In einer Metaanalyse konnten jedoch keine signifikanten Unterschiede im Outcome hinsichtlich Schlaganfall und Tod in Abhängigkeit von der Anwendung eines endoluminalen Shunts (• Abb. 2) oder auch der Wahl des Shuntverfahrens aufgezeigt werden [11].

\section{Perioperatives anästhesiologisches Vorgehen}

Die Zahl der durchgeführten Karotisoperationen hat in den letzten Jahren deutlich zugenommen. Es zeigt sich auch ein Trend zu einer höheren Komorbidität der Patienten (• Tab. 3). Entsprechend der Pathogenese der meisten Karotistenosen weisen Patienten zur TEA eine Vielzahl signifikanter Begleiterkrankungen wie Diabetes mellitus, arterielle Hypertonie und koronare Herzerkrankung (KHK) auf, die in Verbindung mit dem spezifischen operativen Eingriff zu einem verhältnismäßig hohen perioperativen Risiko führen. Im Wesentlichen werden perioperative Morbidität und Mortalität während und nach TEA durch den möglichen Eintritt eines neurologischen Defizits und das Auftreten myokardialer Komplikationen bestimmt.

In der NASCET-Studie wird die Inzidenz eines perioperativen Schlaganfalls mit 5,5\% beziffert; hierbei beläuft sich der Anteil irreversibler bzw. tödlicher Schlaganfälle auf 1,5\%. Die Häufigkeit relevanter perioperativer kardialer Komplikationen wird in der NASCET-Studie mit 3,9\% angegeben. Auch die Langzeitprognose dieser Patienten wird v. a. durch die KHK bestimmt $[16,38,63]$. Das vorrangige Ziel des perioperativen anästhesiologischen Vorgehens muss es daher sein, eine ausreichende zerebrale, aber auch myokardiale Sauerstoffversorgung zu gewährleisten. Gerade diese Feinbalance zwischen ausreichender zerebraler Perfusion und Vermeidung eines erhöhten myokardialen Sauerstoffverbrauchs stellt höchste Ansprüche.

\section{T. Rössel · R.J. Litz · A.R. Heller · T. Koch}

\section{Anästhesie zur Karotischirurgie. Gibt es einen Goldstandard?}

\section{Zusammenfassung}

Die Thrombendarteriektomie (TEA) der A. carotis ist trotz neuer Optionen die Therapie der Wahl in der kurativen Behandlung symptomatischer Karotisstenosen. Da die Patienten während derTEA v. a. durch zerebrale und auch myokardiale Ischämien gefährdet sind, muss das perioperative anästhesiologische Management vorrangig auf die Überwachung und die Sicherstellung der suffizienten Sauerstoffversorgung von Myokard und Gehirn abzielen. Seit Jahren wird kontrovers diskutiert, ob Allgemeinanästhesie- oder Regionalanästhesieverfahren das postoperative Ergebnis verbessern können. Die derzeit vorliegenden wenigen kontrollierten randomisier-

\section{Anesthesia for carotid artery surgery. Is there a gold standard?}

\section{Abstract}

Carotid endarterectomy (CEA) has remained the first choice of treatment in preventing ischemic stroke due to symptomatic stenosis of the internal carotid artery despite other new available options. During CEA patients are first and foremost at risk of cerebral as well as myocardial ischemia, therefore, maintenance of the oxygen supply to the brain and the myocardium is of outstanding importance requiring reliable monitoring of cerebral and cardiac function. The regional versus general anesthesia debate is an age-old one that has brought few definite answers regarding the impact on postoperative outcome of either anesthetic technique. ten Untersuchungen zeigen hinsichtlich harter Outcomeparameter keine signifikanten Vorteile eines der beiden Verfahren. Für die Wahl der Regionalanästhesie kann aufgrund des Evidenzlevels $1^{-}-2^{+}$allenfalls eine GradC-Empfehlung gegeben werden. Die vorliegende Arbeit gibt eine Übersicht über Besonderheiten und Zielsetzungen des anästhesiologischen Managements in der Karotischirurgie.

\section{Schlüsselwörter}

Karotischirurgie · Regionalanästhesie · Allgemeinanästhesie $\cdot$ Komplikationen · Outcome few randomized clinical trials to demonstrate the superiority of either anesthetic technique with respect to outcome parameters. Because the level of evidence in favor of regional anesthesia during CEA can at least be rated between $1^{-}$and $2^{+}$the resulting recommendation is grade $C$. The purpose of the review is to highlight the characteristics and goals of anesthetic management during CEA.

\section{Keywords}

Carotid artery surgery - Regional anesthesia $\cdot$ General anesthesia $\cdot$ Complications · Outcome 

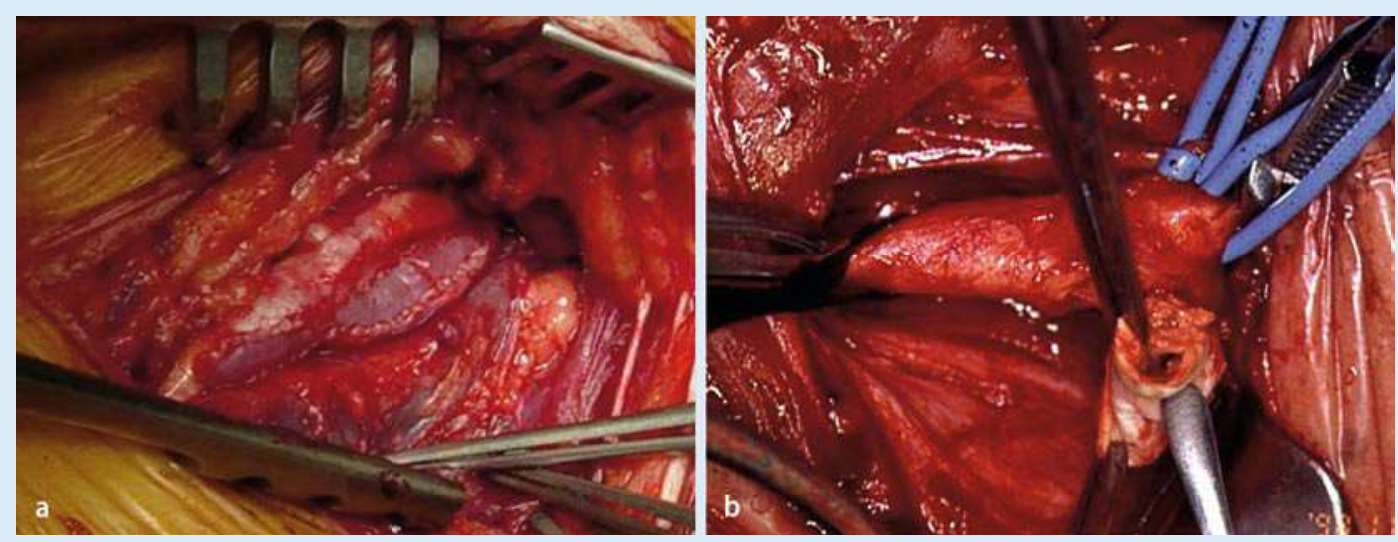

Abb. $1<$ a Operativer Situs nach konventioneller TEA mit Patchplastik und b während Durchführung der Desobliteration im Zuge einer Eversions-TEA

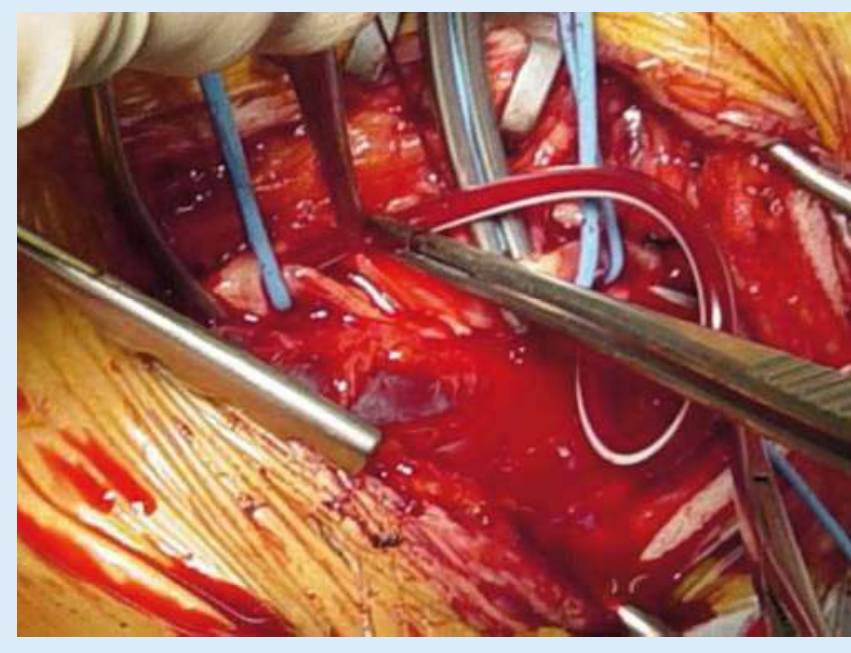

Abb. $2<$ Operativer Situs während der Einbringung eines endoluminalen Shunts im Rahmen einer konventionellen TEA

\section{Prämedikation}

Patienten mit signifikanter ACI-Stenose weisen aufgrund der Vielzahl von Begleiterkrankungen ein hohes perioperatives Risiko auf. Dieser Begleitmorbidität muss bereits bei der Anamneseerhebung und der klinischen Untersuchung Rechnung getragen werden. Gegebenenfalls muss die allgemeine präoperative Diagnostik erweitert werden, um das perioperative Risiko abschätzen und entsprechend minimieren zu können. Neben der Beurteilung des präoperativen neurologischen Status sollte die präoperative Evaluierung der kardiopulmonalen Belastbarkeit daher ebenso obligat sein. Eine Ergänzung der klinischen Befunde durch ein Thoraxröntgen und ein 12-Kanal-EKG ist empfehlenswert. Im Besonderen sollte vor der Durchführung einer Regionalanästhesie eine kontralaterale Phrenikusparese mithilfe des Thoraxröntgens oder alternativ einer Ultraschalluntersuchung der Zwerchfellmotilität ausgeschlossen wer- den. Ein ungenügend eingestellter Hypertonus erhöht das Risiko perioperativer neurologischer und myokardialer Komplikationen. Daher sollte ein unzureichend eingestellter Blutdruck präoperativ optimiert werden. Vor dem Hintergrund möglicher myokardialer Komplikationen ist die Weiterführung einer vorbestehenden antihypertensiven $\mathrm{Me}$ dikation auch am Operationstag empfehlenswert. Bei Patienten mit Diabetes mellitus sollte präoperativ die Normoglykämie angestrebt werden.

\section{Medikamentöse Prämedikation}

Die anxiolytische Prämedikation wurde in den vergangenen Jahren immer wieder kontrovers diskutiert. Die Ansicht, dass die Prämedikation die neurologische $\mathrm{Be}$ urteilbarkeit gefährdet, sollte jedoch kritisch betrachtet werden. Nach unserer Auffassung kann die durch die Prämedikation erreichte Anxiolyse vielmehr als ein wesentlicher Bestandteil der Kardioprotektion betrachtet werden.

\section{Hämodynamik und Kreislaufmonitoring}

Die Inzidenz perioperativer kardialer Komplikationen wird in der NASCETStudie mit 3,9\% angegeben. Der Grund hierfür ist sowohl die begleitende KHK als auch die perioperativ auftretende Blutdruckdysregulation [2, 30, 50, 72]. Die Ursache dieser Dysregulation liegt in der Operationstechnik per se, da sie an einer der wesentlichen Schaltstellen der Blutdruckregulation ansetzt. Mit der intraoperativen Eröffnung des Gefäßes bzw. nach Clamping können die Barorezeptoren des Glomus caroticum einen schlagartigen Druckabfall registrieren; dies kann über neurale Afferenzen eine zentrale Gegenregulation auslösen. Letztlich resultiert diese Gegenregulation in Tachykardie und ausgeprägtem arteriellen Druckanstieg. Die Folge ist ein signifikanter Anstieg des myokardialen Sauerstoffbedarfs. Andererseits können die Manipulation am Glomus caroticum und eine direkte mechanische Reizung des N. vagus zu einer vegetativen 

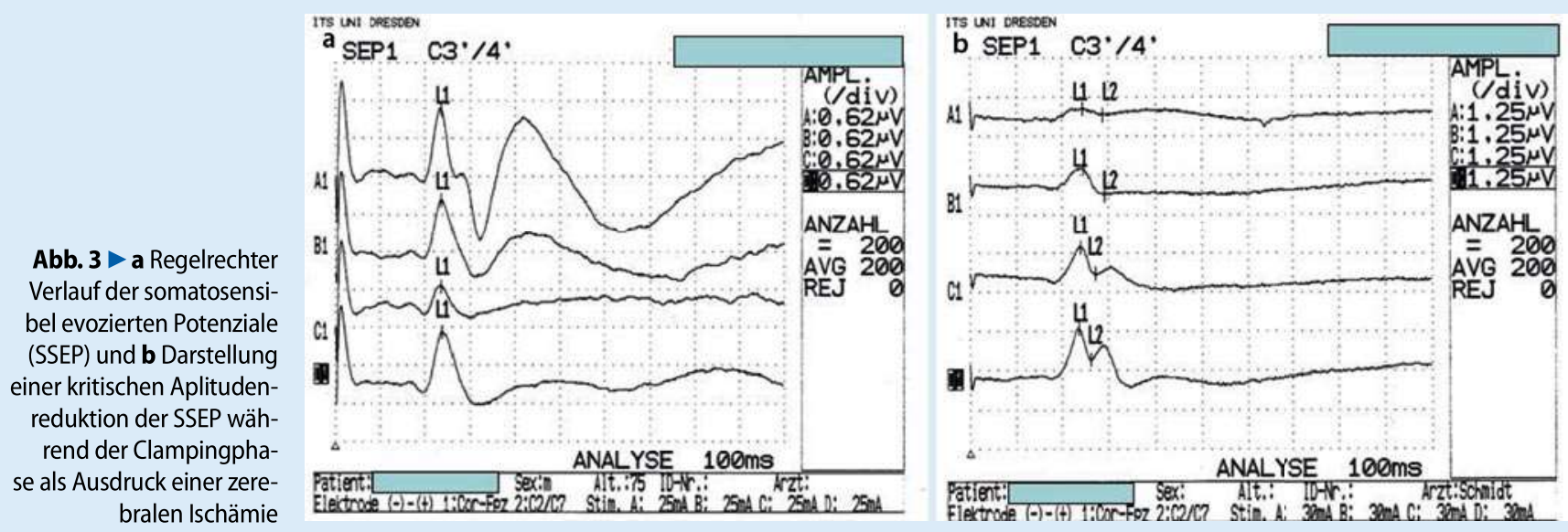

Labilität mit einer ausgeprägten Hypotonie- und Bradykardieneigung führen. Diese bereits intraoperativ eintretenden Störungen der Hämodynamik können über Stunden oder bis zu mehreren Tagen anhalten. Es gilt grundsätzlich, hypotone Blutduckphasen vor dem Hintergrund einer suffizienten zerebralen Perfusion zu vermeiden, ebenso wie hypertensive Phasen, da diese zum Auftreten eines Hyperperfusionssyndroms beitragen können. Ziel des hämodynamischen Managements sollte daher die Einhaltung normotoner und normofrequenter Kreislaufparameter sein; hierbei sollte eine klare Orientierung an den präoperativen Parametern erfolgen. Ein Erzwingen von „Normalwerten“ ohne Berücksichtigung einer möglichen hypertonen Ausgangslage kann das Auftreten einer zerebralen Ischämie während der Clampingphase begünstigen. Die lokale Infiltration von 12 ml Lokalanästhestikum zwischen A. carotis interna und A. carotis externa kann das Auftreten von Bradykardien reduzieren [3]. Die Applikation von Anticholinergika sollte bei vorbestehender KHK nur bei hämodynamisch relevanter und anhaltender Bradykardie erfolgen, da eine mögliche Überkorrektur der Herzfrequenz den myokardialen Sauerstoffverbrauch erhöht. Perioperative Tachykardien können neben der durch den operativen Eingriff hervorgerufenen Dysregulation auch als Folge einer Schmerzoder Stressreaktion auftreten. Aus diesem Grund sollte vor Beginn einer frequenzregulierenden Therapie eine suffiziente Anästhesie/Analgesie gesichert sein. Ist eine Tachykardie auch durch Vertiefung der Anästhesie nicht zu beseitigen, kann die
Therapie mit $\beta$-Blockern indiziert sein; hierbei gestatten kurz wirksame Substanzen eine bessere Steuerung. Während hypertensive Phasen eher unter Regionalanästhesie auftreten, sind hypotensive Phasen unter Allgemeinanästhesie häufiger. Der Grund hierfür liegt zum einen in der durch Anästhetika hervorgerufenen Vasodilatation und ihrer direkt negativ-inotropen Wirkung. Zum anderen aber auch in der Reduktion der Vorlast durch die maschinelle Beatmung. Hypotensive Phasen sollten wegen der Gefahr eines kritischen Abfalls der zerebralen Perfusion während des Clampings unbedingt vermieden werden. Die Therapie der Hypotension umfasst neben einer ausreichenden Volumenzufuhr die Applikation von kurz wirksamen Vasopressoren.

Während in der Clampingphase ein moderat erhöhter Blutdruck die zerebrale Perfusion verbessern kann, sollte eine exzessive Steigerung des systolischen Blutdrucks von $>180$ mmHg aber unbedingt vermieden werden. Ausgeprägte Hypertensionen bergen eher die Gefahr myokardialer Ischämien oder zerebraler Blutungen, die den Nutzen der verbesserten zerebralen Perfusion überwiegt. Die Therapie der intraoperativen Hypertonie sollte aufgrund der guten Steuerbarkeit mithilfe kurz wirksamer Antihypertensiva wie Urapidil erfolgen. Aufgrund des günstigen Effektes auf die koronare Sauerstoffversorgung kann aber auch Nitroglyzerin angewendet werden. Die Komplexität und die Dynamik der möglichen hämodynamischen Veränderungen erfordern ein suffizientes hämodynamisches Monitoring. Hierbei sind neben Standardverfahren wie Pulsoxymetrie und 5-Kanal-
EKG die Mehrkanalanalyse der ST-Strecken sowie die kontinuierlich arterielle Blutdruckmessung sinnvoll. Ein nichtinvasives Monitoring des arteriellen Blutdruckes in mehrminütigen Intervallen wird der Dynamik möglicher hämodynamischer Reaktion nicht gerecht. Ein erweitertes hämodynamisches Monitoring mithilfe des Pulmonalarterienkatheters oder der transösophagealen Echokardiographie gehört sicher nicht zu den Routineverfahren, kann aber bei Hochrisikopatienten in Einzelfällen indiziert sein.

\section{Neuromonitoring}

In der NASCET-Studie wird die Inzidenz eines perioperativen Schlaganfalls mit 5,5\% beziffert; hierbei beläuft sich der Anteil an schweren bzw. tödlichen Schlaganfällen auf 1,5\%. Die ECST-Studie gibt ein Risiko von $7,5 \%$ bzw. $2,8 \%$ an. Ursachen sind sowohl ischämische als auch hämorrhagische Ereignisse. Ischämische Schlaganfälle treten infolge thromboembolischer Ereignisse oder aber nach kritischer Reduktion der zerebralen Perfusion auf. In gesundem Hirngewebe bestimmt die neuronale Aktivität den Metabolismus und damit den zerebralen Blutfluss („cerebral blood flow“, CBF), der im Durchschnitt bei $>40-50 \mathrm{ml} \times 100^{-1} \times \mathrm{min}^{-1}$ liegt. Der Hirnstoffwechsel ist unter normalen Bedingungen ausschließlich auf die aerobe Glykolyse angewiesen. Die geringen zerebralen Glukosedepots erlauben einen anaeroben Stoffwechsel lediglich über einen Zeitraum von etwa 34 min. Daher ist eine konstante Perfusion zur Substratbereitstellung essentiell. Um einen konstanten CBF zu gewährleisten, 


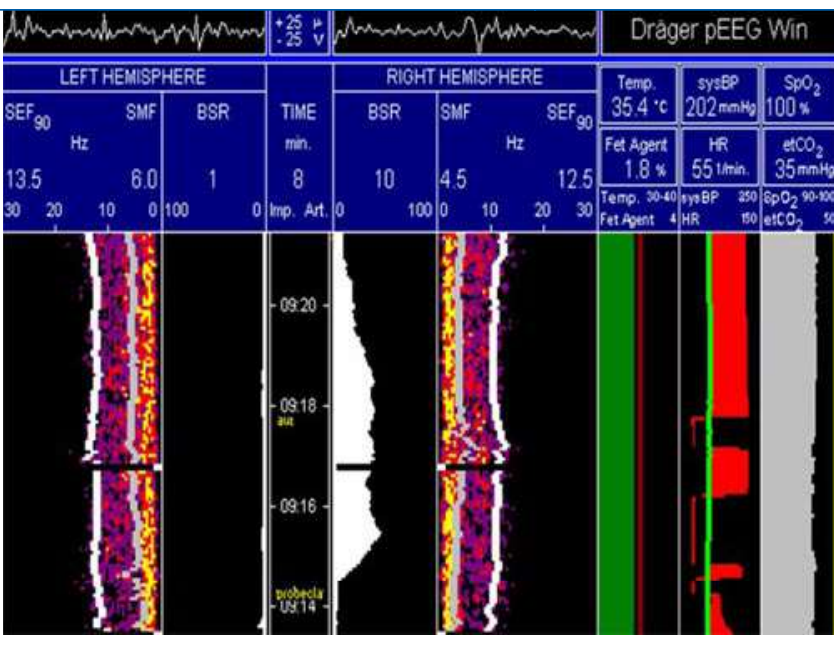

Abb. $4<$ Pathologischer Befund im prozessierten Elektroenzephalogramm unter Allgemeinanästhesie mit einer ischämiebedingten Zunahme der „burst supression ratio" während des Clampings

besitzen die Gefäße des Gehirns innerhalb einer Variationsbreite des mittleren arteriellen Drucks (MAD) von 70-150 mm$\mathrm{Hg}$ die Fähigkeit zur Autoregulation unabhängig vom systemischen Blutdruck. Sinkt der MAD unterhalb dieses Grenzwerts, so nimmt der CBF ab. Daraus resultiert eine globale zerebrale Ischämie. Klinische Symptome sind Übelkeit, Schwindel und Beeinträchtigung der Vigilanz. Im Gegensatz zur globalen Ischämie infolge eines systemischen Blutdruckabfalls treten neurologische Defizite während einer Karotis-TEA meist fokal auf. Die klinische Symptomatik wird durch die Lokalisation der Ischämie bestimmt. Aufgabe des Neuromonitorings während einer Karotis-TEA ist die frühzeitige und zuverlässige Identifikation einer therapierelevanten zerebralen Ischämie durch:

- hohe Sensitivität und Spezifität,

- einfache Bedienung und Messwertinterpretation,

- kontinuierliche Überwachung,

- geringe Artefaktanfälligkeit,

- keine verfahrensbedingten Nebenwirkungen für den Patienten und - geringe zeitliche Latenz.

Als Monitoringverfahren werden bei der TEA elektrophysiologische Verfahren wie die somatosensibel evozierten Potenziale (SSEP) und das Elektroenzephalogramm (EEG), das photometrische Verfahren der Nahinfrarotspektroskopie (,near infrared spectroscopy“, NIRS), aber auch hämodynamisch orientierte Methoden wie die transkranielle Dopplersonographie („transcranial Doppler sonography“, TCD) oder die eher historische Stumpf- druckmessung eingesetzt (• Tab. 4). Eine andere, einfache Möglichkeit des Monitorings der zerebralen Funktion ohne Zwischenschaltung komplexer Datenverarbeitungssysteme ist die klinische Beurteilung des Patienten unter Regionalanästhesie.

\section{Somatosensorisch evozierte Potenziale}

Mit einer Spezifität von $99 \%$ und einer Sensitivität von $83-99 \%$ sind die SSEP ein zuverlässiger Parameter zur Erfassung einer zerebralen Ischämie während der Karotis-TEA (• Abb. 3; [48]). Hierbei werden die SSEP typischerweise nach Stimulation des N. medianus der Gegenseite über dem ipsilateralen Gyrus postcentralis der Operationsseite abgeleitet. Obwohl die Interpretation der SSEP die verschiedenen Einflüsse von Temperatur oder Anästhetika berücksichtigen sollte, konnten zahlreiche Untersuchungen zeigen, dass die SSEP im Vergleich zu anderen elektrophysiologischen Verfahren sowohl am effizientesten eine zerebrale Ischämie erfassen können und auch am robustesten gegenüber externen Einflüssen sind [29, 47, 75].

Zur Identifikation einer Minderperfusion werden folgende Parameter herangezogen:

- Latenzen und Amplituden des kortikalen Potenzials ( $20 / \mathrm{P}_{25}$ ),

- Latenz des zervikalen Potenzials

(N14) sowie

- zentrale Überleitungszeit zwischen

$\mathrm{N}_{13}$ und $\mathrm{N}_{20} \mathrm{O}$ (CCT).
Über die Wertung und die Interventionsgrenzen besteht jedoch keine Einigkeit. Die bisherige Meinung, dass erst eine Abnahme des N2o-Potenzials um mehr als 50\% und ein Anstieg der Latenz um mehr als 10\% die Indikation zur Shunteinlage darstellt, muss anhand neuerer Datenlage kritisch interpretiert werden. Sbarigia et al. konnten schon 2001 am wachen Patienten zeigen, dass bereits eine Abnahme des N2O-Potenzials um 3040\% mit einem neurologischen Defizit korrelierte [79].

\section{Elektroenzephalogramm}

Das EEG stellt eine Aufzeichnung der spontanen elektrischen Aktivität des zerebralen Kortex dar. Die Wellen des sog. Roh-EEG (rEEG) werden hierbei in die Frequenzbereiche $\alpha(8-13 \mathrm{~Hz}), \beta(>13 \mathrm{~Hz})$, $\theta(4-8 \mathrm{~Hz})$ und $\delta(0,5-4 \mathrm{~Hz})$ unterteilt. Da die Interpretation eines solchen EEG jedoch erhebliche Erfahrung voraussetzt, wurde das sog. prozessierte-EEG (pEEG; - Abb.4) eingeführt. Hierbei wird das ursprüngliche EEG-Signal mithilfe der Fast-Fourier-Transformation auf seine Frequenzanteile hin analysiert. Das Resultat ermöglicht eine relativ einfache Interpretation ohne wesentlichen Informationsverlust für den Anwender. Zerebrale Ischämien können über Frequenzverschiebungen in Richtung eines langsameren Wellenmusters detektiert werden. Diese pEEG-Veränderungen sind im Allgemeinen etwa 1 min nach Ischämiebeginn $\mathrm{zu}$ verzeichnen und treten in den meisten Fällen asymmetrisch auf. Als Interventionsgrenze wird eine Abnahme der spektralen Eckfrequenz (SEF) von $\geq 50 \%$ angegeben $[7,70,85]$. Im Gegensatz zu den SSEP unterliegt das $\mathrm{pEEG}$ jedoch stärker externen Einflüssen und muss aus diesem Grund als weniger robust bezeichnet werden. McFarland et al. konnten zeigen, dass die Spezifität des pEEG mit $98,5 \%$ ähnlich der von SSEP ist, jedoch wird die Sensitivität des pEEG mit lediglich 55\% angegeben [54].

\section{Nahinfrarotspektroskopie}

Das Prinzip der NIRS besteht in der Messung der Konzentrationen von Oxyhämoglobin, Desoxyhämoglobin und oxidiertem Zytochrom aa ${ }_{3}$ im durchstrahlten Gewebe. Hierzu wird Licht mit einer 
Wellenlänge von 700-1000 nm durch eine auf der Haut angebrachten Optode durch die Kutis, die Subkutis und die Kalotte bis ins unmittelbar angrenzende Hirngewebe gesendet. Die Abschwächung des Lichtstrahls wird von einer Empfängeroptode erfasst. Anschließend erfolgt die rechnergestützte Berechnung der regionalen Sauerstoffsättigung des Hirngewebes. Der theoretische Vorteil dieser Methode besteht in der frühzeitigen Erfassung zellulärer Oxygenierungsstörungen, noch bevor eine neuronale Funktionseinschränkung sichtbar wird. Prospektiv randomisierte Untersuchungen, die diese theoretische Überlegung zuverlässig stützen, liegen jedoch derzeit nur in unzureichendem $\mathrm{Maß}$ vor. Aus diesem Grund sollte die Anwendung der NIRS als alleiniges Monitoringverfahren zur Karotis-TEA kritisch betrachtet werden. Eingebettet in ein Neuromonitoringkonzept, wie dies von Michel et al. vorgestellt wurde, kann die NIRS jedoch wertvolle Zusatzinformationen liefern und damit zur Sicherheit des Patienten beitragen [59].

\section{Transkranielle Dopplersonographie}

Die TCD nutzt die Geschwindigkeit der korpuskulären Blutbestandteile in der A. cerebri media, um Rückschlüsse auf die kortikale Durchblutung zu ziehen (• Abb.5). Aus dem reflektierten Frequenzspektrum lassen sich systolische $\left(\mathrm{V}_{\text {syst }}\right)$, mittlere $\left(\mathrm{V}_{\text {mean }}\right)$ und diastolische $\left(V_{\text {dia }}\right)$ Blutflussgeschwindigkeiten ableiten. Der Normbereich für $\mathrm{V}_{\text {mean }}$ der ACI ist dabei mit $38-86 \mathrm{~cm} / \mathrm{s}$ relativ breit, sodass es sinnvoll erscheint, die gemessenen TCD-Werte während einer Karotis-TEA immer im Verlauf zu beurteilen. Uneinigkeit herrscht über die relevanten „Cut-off“-Werte zur Detektion einer kritischen zerebralen Perfusionseinschränkung. Schwere Ischämien sollen bei einem $\mathrm{V}_{\text {mean }}$-Abfall von mehr 85\% des Ausgangswerts resultieren, während ein Abfall von weniger als 60\% des Ausgangswerts zu keiner klinisch relevanten Ischämie führen soll $[14,46]$. Ein Defizit dieses Verfahrens ist, dass die Anwendung des TCD keine Rückschlüsse auf den zerebralen Funktionszustand zulässt. Ebenfalls von Nachteil ist, dass bei 10-40\% der Patienten kein Flowsignal ableitbar ist. Somit stellt die TCD allenfalls ein supportives Verfah- ren dar, das für den alleinigen Einsatz zur Detektion einer zerebralen Ischämie nicht geeignet ist $[23,33]$.

\section{Stumpfdruckmessung}

Mehrere Studien konnten zeigen, dass der Stumpfdruck nur schlecht mit dem zerebralen Perfusionsdruck korreliert. Entsprechend lassen sich intraoperative neurologische Defizite mit der Stumpfdruckmessung nur unzureichend vorhersagen $[9,45]$. Obwohl diese Mängel durchaus bekannt sind, ist die Anwendung der Stumpfdruckmessung in vielen Kliniken das Routineverfahren zur Detektion eines zerebralen Perfusionsdefizits. Aufgrund der aktuellen Studienlage besteht jedoch in der alleinigen Anwendung der Stumpfdruckmessung kein suffizientes Monitoringverfahren.

\section{Neuromonitoring unter Regionalanästhesie}

Das Monitoring der neurologischen Funktion unter Regionalanästhesie unterscheidet sich grundlegend von den unter Allgemeinanästhesie eingesetzten apparativen Verfahren. Die ständige Kommunikation mit dem Patienten und die Kontrolle der motorischen Funktionen mit einem Gummiquietschtier in der kontralateralen Hand ( $\bullet$ Abb.6) machen das frühzeitige Erkennen neurologischer Defizite relativ einfach. Da auf die zusätzliche Zwischenschaltung eines technischen Verfahrens verzichtet wird, kann dieses Monitoring als störungsfrei angesehen werden. Nach Ansicht einiger Autoren sind wache Patienten der "Goldstandard“ für die Überwachung der zerebralen Funktion während der KarotisTEA. Hans u. Jareunpoon zeigten in einer prospektiven Untersuchung an 314 Patienten, das sowohl EEG als auch Stumpfdruckmessung hinsichtlich Spezifität und Sensitivität dem Monitoring des wachen Patienten unter Regionalanästhesie unterlegen sind [37]. Zu ähnlichen Ergebnissen kamen Schneemilch et al. unter Anwendung von SSEP [80]. Diese Einschätzung trifft sicher für kooperative Patienten und komplikationslose Operationsabläufe zu. Diese Art des Neuromonitorings kann jedoch durch eine Vielzahl äußerer Faktoren erheblich eingeschränkt werden. Bei Patienten mit neurologischen oder psych- iatrischen Grunderkrankungen kann die zuverlässige neurologische Beurteilung erheblich erschwert oder sogar unmöglich sein. Daher muss die Patientencompliance im Vorfeld abgeklärt werden.

\section{Antikoagulation}

Die Dauertherapie mit Thrombozytenaggregationshemmern stellt einen Eckpfeiler der nichtoperativen Therapie bei Patienten mit ACI-Stenose dar. Acetylsalicylsäure und Clopidogrel sind dabei die beiden am häufigsten in Deutschland verwendeten Wirkstoffe. Während Clopidogrel die Adenosindiphosphat (ADP) vermittelte Thrombozytenaggregation hemmt, wird die Wirkung von Acetylsalicylsäure über eine Hemmung der Zyklooxygenase vermittelt. In Anbetracht der gegenwärtigen Studienlage ist eine präoperative Unterbrechung dieser Therapie nicht angeraten. Die Kombination von Acetylsalicylsäure und Clopidogrel ist jedoch nicht zu empfehlen, da einerseits das Blutungsrisiko schwer kalkulierbar ist und andererseits keine signifikante Verbesserung des neurologischen Outcomes durch die Anwendung beider Substanzen nachgewiesen werden konnte [31].

Intraoperativ besteht während der Clampingphase, aber auch durch die Einbringung eines endoluminalen Shunts die Gefahr der Bildung von Appositionsthromben. Zur Minimierung dieses Risikos wird daher vor Beginn der Clampingphase meist unfraktioniertes Heparin appliziert. Der Heparineffekt kann mithilfe der ,activated clotting time“ (ACT) als „Point-of-care“-Verfahren bettseitig überwacht werden [20]. Eine Antagonisierung des Heparineffektes durch Protamin sollte aufgrund der möglichen thromboembolischen Komplikationen nicht routinemäßig, sondern immer nach individueller Einschätzung des Blutungsrisikos erfolgen.

\section{Anästhesieverfahren}

Die TEA der A. carotis interna wird sowohl unter Regionalanästhesie als auch Allgemeinanästhesie durchgeführt. Bislang dominieren in Deutschland die Allgemeinanästhesieverfahren eindeutig, der Anteil an Regionalanästhesietechniken nimmt aber kontinuierlich zu (• Abb. 7). 

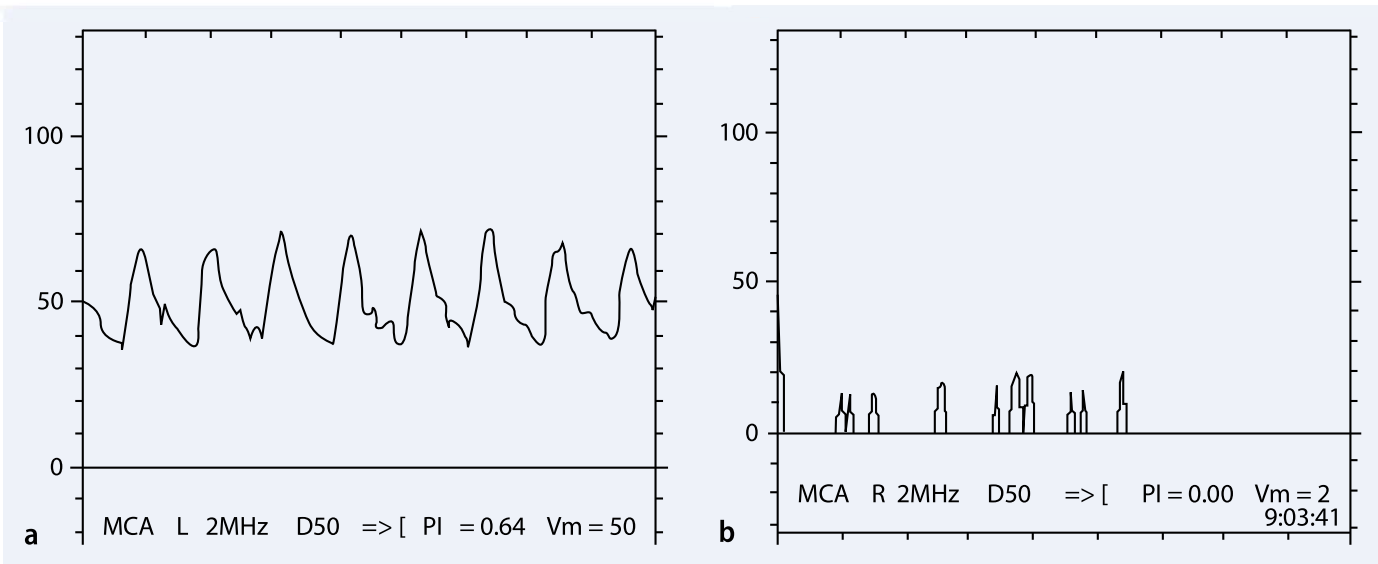

Abb. $5<$ a Transkranielle Dopplersonographie (TCD) mit regelrechter Flussgeschwindigkeit in der A. cerebri media und $\mathbf{b}$ Darstellung einer TCD-Untersuchung mit kritischer Reduktion der Flussgeschwindigkeit während der Clampingphase

Unabhängig vom gewählten Anästhesieverfahren muss jedoch sichergestellt werden, dass der Anästhesist Vor- und Nachteile des jeweiligen Verfahrens sowie dessen Risiken kennt und das Anästhesieverfahren auf den individuellen Patienten ausrichtet.

\section{Regionalanästhesieverfahren}

Nach Angaben der Deutschen Gesellschaft für Gefäßchirurgie liegt der Anteil der in Regionalanästhesie durchgeführten Operationen zur Karotischirurgie gegenwärtig bei $<20 \%$. Demnach kommt der Regionalanästhesie in der Karotischirurgie in Deutschland eine vergleichsweise geringe Bedeutung zu. Der wesentliche Vorteil dieses Verfahrens ist die einfache und schnelle neurologische Beurteilbarkeit. Daher erscheint dieses Anästhesieverfahren vorteilhaft bei Anwendung der selektiven Shuntanlage. Die fehlende Neuroprotektion und die erschwerte bis unmögliche Anwendung bei unkooperativen Patienten müssen jedoch als Nachteile gewertet werden. Entsprechend des operativen Situs sollte bei Durchführung einer Regionalanästhesie die Ausdehnung der sensorischen Blockade die Dermatome $\mathrm{C}_{2}-\mathrm{C}_{4}$ beinhalten. Aufgrund der komplexen Innervation des Operationssitus durch Zervikalund auch Hirnnerven ist jedoch in den meisten Fällen eine intraoperative Supplementierung durch den Operateur erforderlich, da gerade die Hirnnervenanteile nicht ausreichend anästhesiert werden [19]. Somit stehen für die Karotischirurgie grundsätzlich die folgenden 3 Verfahren der Regionalanästhesie zur Auswahl:
- zervikale Epiduralanästhesie,

- oberflächliche Zervikalblockade und

- tiefer Zervikalblock nach Moore [61] oder als interskalenäre Blockade nach Winnie [9o].

\section{Epiduralanästhesie}

In Deutschland nimmt die Epiduralanästhesie zur Karotis-TEA im Gegensatz zu Frankreich oder Australien lediglich eine Randstellung ein; dies mag an der zumeist begleitenden Therapie mit Thrombozytenaggregationshemmern liegen. Die Punktion erfolgt in Höhe C6/ $\mathrm{C}_{7}$, anschließend wird ein Katheter ca. $4 \mathrm{~cm}$ in den Epiduralraum eingeführt. Seraglio u. Campostella zeigten, dass die Analgesiequalität von Epiduralanästhesie und Zervikalblockade gleichwertig war [81]. Jedoch verursachte die Epiduralanästhesie häufiger interventionspflichtige Blutdruckabfälle. Für zervikale Epiduraltechniken scheint das Risiko ernsthafter Komplikationen nicht höher zu sein als für lumbale Techniken. Allerdings liegen keine ausreichenden Erfahrungen für Patienten mit karotischirurgischen Eingriffen vor, sodass zur Abschätzung des Risikos Erfahrungen aus anderen Bereichen herangezogen werden müssen. Patienten zur Karotisoperation haben häufig eine Begleittherapie mit Thrombozytenaggregationshemmern und somit eine Kontraindikation für die Epiduralanästhesie. Da bei hochgradigen Stenosen die perioperative Unterbrechung der antikoagulatorischen Therapie nicht empfohlen wird, öffnet sich kaum ein zeitliches Fenster, das die Anlage eines rückenmarknahen Analgesieverfahrens bei vertretbarem Risiko erlauben würde [6o].

\section{Oberflächliche Zervikalblockade}

Die oberflächliche Zervikalblockade wird durchgeführt, um die sensiblen Äste des Plexus cervicalis zu anästhesieren. Die Punktionsstelle befindet sich in der Mitte der Verbindungslinie zwischen Mastoid und Processus transversalis des sechsten Halswirbelkörpers am Hinterrand des M. sternocleidomastoideus. Diese Lokalisation entspricht dem Punctum nervosum, dem Austritt des oberflächlichen Plexus cervicalis. Die Kanüle wird unter dem Hinterrand des M. sternocleidomastoideus durch die superfiziale Halsfaszie vorgeschoben, ohne die tiefe Halsfaszie $\mathrm{zu}$ perforieren. Es werden $5 \mathrm{ml}$ Lokalanästhetikum injiziert. Danach werden von derselben Einstichstelle ausgehend zunächst nach kranial und dann nach kaudal jeweils erneut $5 \mathrm{ml}$ Lokalanästhetikum (z. B. 1\%ges Mepivacain) appliziert. Hinsichtlich der analgetischen Effektivität ist diese oberflächliche mit der tiefen Zervikalblockade vergleichbar [65, 83], da sich das Lokalanästhetikum nach Durchführung einer oberflächlichen Zervikalblockade entlang der Nervenbahnen über die Fasziengrenzen hinweg in die Tiefe ausbreitet [66].

\section{Tiefe Zervikalblockade}

Die am häufigsten verwendete Methode zur Blockade des Plexus cervicalis profundus ist die klassische Technik nach Moore. Hierzu müssen 3 Infiltrationspunkte identifiziert werden, die auf einer Verbindungslinie zwischen dem Processus mastoideus und dem Querfortsatz des sechsten Halswirbelkörpers lokalisiert sind. Der Querfortsatz des vierten Halswirbelkörpers kann meist am unteren Drittel dieser Verbindungslinie in Höhe 
des Krikoids getastet werden. Der Processus transversalis des zweiten Zervikalwirbels befindet sich auf dieser Linie ca. 1,5-2 cm kaudal des Mastoids. $\mathrm{C}_{3}$ liegt genau zwischen den beiden zuvor genannten Punkten. Eine immobile 22-G-Kanüle wird senkrecht zur Haut und leicht kaudal geneigt vorgeschoben, bis in einer Tiefe von $1,5-3,5 \mathrm{~cm}$ je nach Halsumfang Kontakt mit dem korrespondierenden Processus transversalis hergestellt werden kann. Nach Zurückziehen der Kanüle um 1$2 \mathrm{~mm}$ werden 5-8 $\mathrm{ml}$ Lokalanästhetikum (z. B. 0,5\%iges Ropivacain) langsam injiziert. Dieser Vorgang wird analog bei $\mathrm{C}_{3}$ und $\mathrm{C}_{4}$ wiederholt.

Als alternative Technik zur Blockade nach Moore [61] bietet sich die Einzelinjektion nach Winnie [90] an. Diese Technik unterscheidet sich insofern von der klassischen Interskalenusblockade, als nicht in Höhe von C6 der Plexus brachialis, sondern in Höhe von $\mathrm{C}_{4} / \mathrm{C}_{5}$ die kaudalen Anteile des Plexus cervicalis anästhesiert werden. Die Punktion kann dabei mit oder ohne periphere elektrische Nervenstimulation (PNS) ausgeführt werden. Inwieweit die PNS jedoch den Erfolg zervikaler Blockaden verbessert, wird kontrovers beurteilt. So fanden Mehta u. Juneja eine Steigerung des Blockadeerfolgs unter Verwendung eines Nervenstimulators [56]. Merle et al. verglichen den klassischen tiefen Zervikalblock mit der Einzelinjektionstechnik unter PNS. Sie fanden eine vergleichbare Effektivität beider Blockaden, vermuteten aber bei der Einzelinjektionstechnik eine geringere systemische Lokalanästhetikaabsorption [57].

Die Blockade des Plexus cervicalis profundus kann zu einer Vielzahl von Komplikationen führen. De Sousa et al. konnten zeigen, dass die kombinierte Zervikalblockade, bestehend aus tiefem und oberflächlichem Zervikalblock, zu deutlich mehr punktionsbedingten Komplikationen führte als die oberflächliche Zervikalblockade [21]. Zu ähnlichen Ergebnissen kamen Pandit et al. [67]. Sie verglichen 69 Studien mit insgesamt 7558 kombinierten und 2533 oberflächlichen Blockaden des Plexus cervicalis und fanden unter Anwendung der oberflächlichen Zervikalblockade signifikant weniger punktionsbedingte Komplikationen. Davis et al. berichten über versehentliche

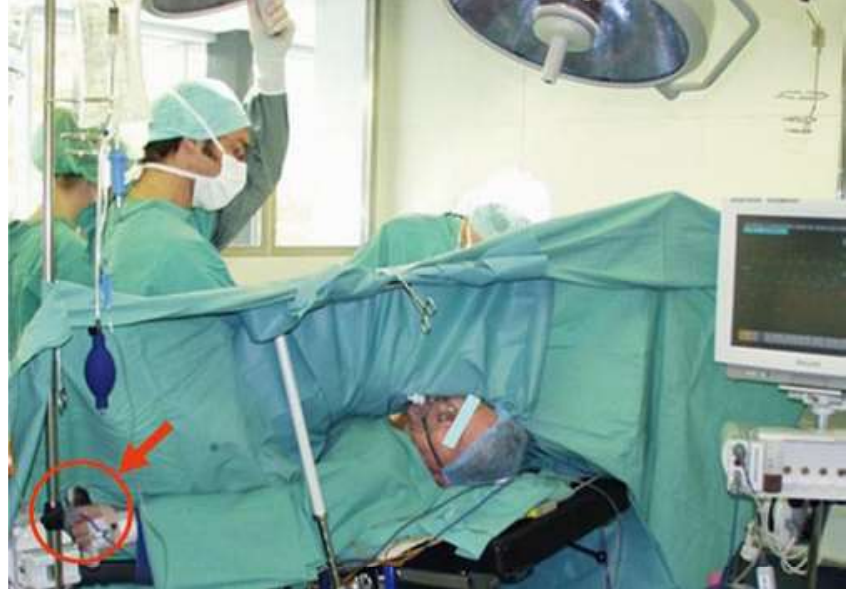

Abb. 6 $>$ Neuromonitoring unter Regionalanästhesie. Die Kommunikation mit dem Patienten und die Kontrolle der kontralateralen motorischen Funktion (Markierung, Gummiquietschtier) erlauben eine schnelle und zuverlässige Erfassung des neurologischen Status

Gefäßpunktionen in bis zu 66\% der Fälle; die meistens jedoch ohne Folgen blieben [19]. Allerdings zeigten diese Erfahrungen auch, dass wiederholte Aspiration während der Injektion keinen zuverlässigen Schutz vor Fehlinjektion bietet. Berichte über Krampfanfälle bei Injektion geringer Dosen von Lokalanästhetika waren am ehesten auf versehentliche intravasale Injektion von Lokalanästhetika während der Supplementierung bei insuffizienten Blockaden zurückzuführen [82].

Erste Erfahrungen mit der Durchführung einer ultraschallgestützten Zervikalblockade zeigen, dass hier in der $\mathrm{Zu}$ kunft eine neue Dimension der Sicherheit erreicht werden könnte, da bereits die Injektion und die Ausbreitung von Volumina $<1 \mathrm{ml}$ visuell zuverlässig verfolgt werden kann [74]. Darüber hinaus kann eine sichere Blockade mit einem Minimum an Lokalanästhetikadosis gewährleistet werden.

\section{Allgemeinanästhesieverfahren}

In Deutschland stellt die Allgemeinanästhesie mit einem Anteil $>80 \%$ das am häufigsten genutzte Anästhesieverfahren zur Karotis-TEA dar. Diese Technik gewährleistet neben der für den Patienten schmerz- und stressfreien Operation ein bewegungsfreies Operationsgebiet und damit optimale Operationsbedingungen. Ein weiterer wichtiger Vorteil der Allgemeinanästhesie besteht in den zerebroprotektiven Eigenschaften der verwendeten Medikamente. Ein Nachteil ist die verstärkte Hypotonieneigung insbesondere während der Einleitungsphase. Ein weiterer Nachteil ist, dass alle unter Allgem- einanästhesie angewendeten apparativen Verfahren des intraoperativen Neuromonitorings in ihrer Sensitivität und Spezifität dem Monitoring des wachen Patienten unterlegen sind. Somit ist die endgültige neurologische Erfolgskontrolle erst nach Beendigung der Narkose möglich.

Im Rahmen der Durchführung einer Allgemeinanästhesie hat die Narkoseeinleitung eine besondere Relevanz. Insbesondere die Aufrechterhaltung einer suffizienten Hämodynamik ist vor dem Hintergrund einer ausreichenden zerebralen und koronaren Perfusion von entscheidender Bedeutung. Die Narkoseeinleitung ist grundsätzlich mit jedem Hypnotikum möglich. Etomidat scheint, bedingt durch sein hämodynamisches Profil, gerade bei Patienten mit erhöhtem kardialen Risiko Vorteile zu bieten. Es sollte jedoch beachtet werden, dass die Anwendung von Etomidat die Amplitude des somatosensorisch evozierten kortikalen Summenaktionspotenzials erhöht.

Thiopental und Propofol stellen derzeit die am häufigsten verwendeten Anästhetika zur Induktion einer Allgemeinanästhesie dar. Der zerebroprotektive Effekt von Thiopental ist dabei am besten untersucht. In der Einleitungsphase tritt dieser zerebroprotektive Effekt jedoch in den Hintergrund, da hierbei die Gefahr für den Patienten eher in einer kritischen Reduktion der koronaren Perfusion zu suchen ist. Für Propofol konnten ebenfalls zerebroprotektive Effekte nachgewiesen werden, gleichzeitig zeichnet es sich jedoch durch eine gute Steuerbarkeit und eine für den Patienten angenehme Einschlaf- und Aufwachphase aus. Godet et al. [34] verglichen in einer randomisier- 


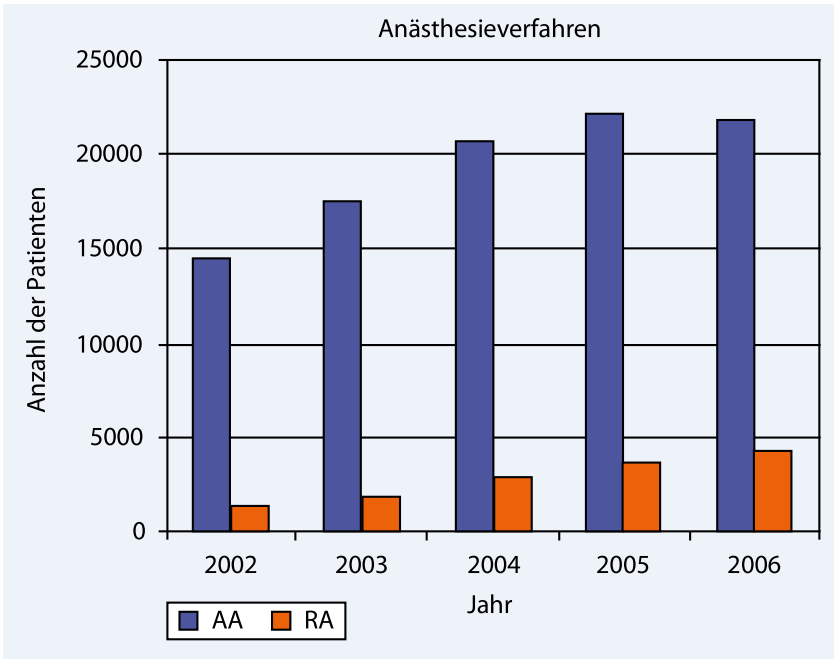

Abb. $7<$ Verteilung der Anästhesieverfahren. (Bundesgeschäftsstelle Qualitätssicherung [12])

ten Arbeit verschiedene Regime der Anästhesieführung hinsichtlich hämodynamischer Effekte sowie die Qualität der Narkoseeinleitung und -ausleitung. Diese Arbeit zeigte, dass sowohl die inhalative Einleitung mit Sevofluran als auch die Bolusapplikation von Propofol und die „target controlled infusion“ (TCI) mit Propofol während der Narkoseeinleitung zu einem Abfall des systemischen Blutdrucks führt, der jedoch in der Sevofluran-Gruppe am geringsten ausgeprägt war. Allerdings wurden die Intubationsbedingungen in der Sevofluran-Gruppe im Vergleich zur Anwendung von Propofol als schlechter eingestuft.

Während der Narkoseeinleitung stellt die Laryngoskopie in Verbindung mit der Intubation den stärksten Schmerzreiz dar. Die in der Folge ausgelöste Stressreaktion kann durch Steigerung des myokardialen Sauerstoffbedarfs bei entsprechend prädisponierten Patienten eine kardiale Ischämie hervorrufen. Daher ist neben einer ausreichenden Hypnosetiefe v. a. eine suffiziente Analgesie entscheidend. Die hierbei am häufigsten verwendeten Analgetika sind Fentanyl, Sufentanil und Remifentanil. Ein Vergleich dieser Substanzen erbrachte keine signifikanten Unterschiede hinsichtlich der Inzidenz an therapiepflichtigen hypotensiven Phasen. Mouren et al. konnten im Vergleich zwischen Remifentail und Sufentanil zeigen, dass die durch die Intubation bedingte Stressantwort durch Remifentanil suffizienter unterdrückt wird [62].

Vor dem Hintergrund einer ausgeprägten Neigung zu Bradykardien sollte jedoch auf die Bolusapplikation von Re- mifentanil verzichtet werden. Die Relaxierung kann mit jedem klinisch üblichen Muskelrelaxans erfolgen; hierbei sollte der Einsatz von Succinylcholin bei Patienten mit chronisch denervierter Muskulatur vermieden werden. Die favorisierte Methode zur Sicherung des Atemwegs während einer Karotis-TEA ist die endotracheale Intubation. Der Vorteil besteht ohne Zweifel in einem zuverlässigen Aspirationsschutz und in der Möglichkeit einer suffizienten mechanischen Ventilation. Im Gegensatz dazu zeigten Marietta et al., dass durch die Anwendung der Larynxmaske zur Sicherung der Atemwege die Inzidenz an tachykarden und hypertensiven Kreislaufreaktionen signifikant reduziert werden kann [49]. Allerdings bietet die Larynxmaske keinen absolut sicheren Aspirationsschutz. Außerdem kann in seltenen Fällen die mechanische Ventilation erschwert sein.

Die Aufrechterhaltung der Narkose kann sowohl mithilfe volatiler als auch intravenöser Anästhestetika erfolgen. Während Kozmary et al. [44] keine erhöhte Inzidenz an intra- und postoperativen myokardialen Ischämien unter Verwendung von Lachgas feststellen konnten, zeigten Badner et al. [6], dass der Einsatz von Lachgas mit einer erhöhten Inzidenz an postoperativen myokardialen Ischämien einhergehen kann. Um postoperativ eine zeitnahe neurologische Beurteilbarkeit zu gewährleisten, sollte das Verfahren so gewählt werden, dass eine rasche Anästhesieausleitung möglich ist. Wegen ihrer guten Steuerbarkeit und ihrer zerebroprotektiven Effekte werden daher häufig volatile Anästhetika als Bestandteil der Nar- kose verwendet. Eine gemeinsame Wirkung aller volatilen Anästhetika besteht in einer Reduktion des Hirnstoffwechsels. Dieser zerebroprotektive Effekt ist jedoch unterschiedlich ausgeprägt und scheint für Isofluran am bedeutsamsten. Messick et al. [58] ermittelten unter EEG-Monitoring den minimalen $\mathrm{CBF}$, der ohne pathologische Veränderungen toleriert wird. Dabei wies Isofluran mit einem minimalen $\mathrm{CBF}$ von $10 \mathrm{ml} / 100 \mathrm{~g}$ und min einen signifikant niedrigeren Wert auf als Halothan mit $18 \mathrm{ml} / 100 \mathrm{~g}$ und min. McCulloch et al. [53] konnten zeigen, dass Sevofluran hinsichtlich der zerebralen Perfusion eine Besonderheit besitzt. Sie verglichen die Veränderungen in der zerebralen Hämodynamik bei Sevofluran- sowie Propofolgabe und postulierten ein zerebrales Steal-Phänomen für die Anwendung von Sevofluran. Welche klinische Bedeutung diese Entdeckung hat und inwieweit sie das neurologische Outcome beeinflusst, kann jedoch zum gegenwärtigen Zeitpunkt noch nicht beantwortet werden.

Umbrain et al. [86] untersuchten den Einsatz von Isofluran, Sevofluran und Desfluran hinsichtlich des Aufwachverhaltens und der perioperativen Hämodynamik. Diese Studie zeigte, dass die Anwendung von Isofluran mit einer verlängerten Aufwachphase assoziiert ist. Keine Unterschiede zeigten sich postoperativ in der Inzidenz von Übelkeit und Erbrechen sowie des „shivering“. Ebenfalls keine Veränderung zeigte das hämodynamische Monitoring anhand von ST-Analyse und Herzindex. Mit Sevofluran und Desfluran, aber auch mit Propofol stehen Medikamente mit einer guten Steuerbarkeit zur Verfügung, die eine rasche neurologische Beurteilbarkeit erlauben.

Die Art des verwendeten Opiats beeinflusst die neurologische Beurteilbarkeit ebenfalls [89]. Am schnellsten ist die neurologische Beurteilbarkeit nach Remifentanil zu erreichen. In Bezug auf hämodynamische Stabilität oder postoperative Übelkeit zeigten sich keine Unterschiede zwischen Fentanyl, Sufentanil und Remifentanil [24, 62]. De Castro et al. [22] untersuchten die Hämodynamik in Abhängigkeit von der Art der Remifentanilapplikation. Diese Arbeit konnte verdeutlichen, dass die TCI im Vergleich zur gewichtsadaptierten kontinuierlichen Gabe 
mit einer stabileren Hämodynamik vergesellschaftet war. Die balancierte Anästhesie mit Isofluran und Fentanyl zeigte im Vergleich mit einer total intravenösen Anästhesie (TIVA) aus Remifentanil und Propofol eine geringere Inzidenz myokardialer Wandbewegungsstörungen, allerdings ohne postoperative EKG-Veränderungen oder erhöhte Troponinspiegel [39] In dieser Untersuchung war jedoch postoperativ in der TIVA-Gruppe eine erhöhte Hypertonieneigung zu beobachten.

Die Suche nach dem optimalen Beatmungsregime war lange Zeit Gegenstand der Diskussion. So verursacht eine Hyperkapnie, bedingt durch die generalisierte zerebrale Vasodilatation, eine Erhöhung der zerebralen Durchblutung. Gleichzeitig kommt es jedoch zu einer Umverteilung des Blutes von ischämischen Hirnarealen hin zu $\mathrm{CO}_{2}$ reagiblen, nichtischämischen Arealen. Dies führt sekundär zu einer Abnahme der Perfusion der ohnehin schon ischämischen Gebiete im Sinne eines Steal-Phänomens. Die Hypokapnie hingegen kann zwar zu einer Verbesserung der Perfusion in den ischämischen Gebieten führen, jedoch kommt es durch die damit verbundene Linksverschiebung der Sauerstoffbindungskurve zu einer verringerten Sauerstoffabgabe an das Hirngewebe. Es liegen gegenwärtig aber keine ausreichend großen Untersuchungen für die Karotis-TEA vor, die klare evidenzbasierte Rückschlüsse erlauben würden, sodass die Aufrechterhaltung einer Normo-

Tab. 5 Prozentuale Komplikationsrate der Karotis-TEA in Deutschland. (Bundesgeschäftsstelle Qualitätssicherung [12])

\begin{tabular}{|llllll|}
\hline Komplikation & \multicolumn{2}{l}{ Erfassungsjahr } & & & \\
& $\mathbf{2 0 0 2}$ & $\mathbf{2 0 0 3}$ & $\mathbf{2 0 0 4}$ & $\mathbf{2 0 0 5}$ & $\mathbf{2 0 0 6}$ \\
\hline Tod [\%] & 0,97 & 0,93 & 0,93 & 0,88 & 0,94 \\
\hline Pulmonal [\%] & 0,94 & 0,73 & 086 & 0,73 & 0,92 \\
\hline Kardial [\%] & 2,36 & 1,79 & 1,86 & 1,86 & 1,89 \\
\hline Nervenläsion [\%] & 1,99 & 1,90 & 1,55 & 1,51 & 1,49 \\
\hline Nachblutung [\%] & 2,65 & 2,55 & 2,79 & 2,95 & 2,53 \\
\hline Wundinfektion [\%] & 0,24 & 0,24 & 0,27 & 0,19 & 0,19 \\
\hline
\end{tabular}

kapnie und einer Normoxämie derzeit am sinnvollsten erscheint.

\section{Postoperativer Verlauf}

Im intra- und postoperativen Verlauf können eine Vielzahl an möglichen Komplikationen wie Blutdruckdysregulation und myokardiale Ischämie sowie neurologische Komplikationen auftreten (- Tab. 5).

Postoperative Störungen der Blutdruckregulation sind in vielen Fällen eine Folge des operativen Eingriffs selbst. Die manipulativen Veränderungen im Bereich des Glomus caroticum verursachen postoperativ häufig eine hypertensive Blutdruckdysregulation. Dabei konnte gezeigt werden, dass die Eversions-TEA im Vergleich zur konventionellen TEA postoperativ signifikant häufiger zu hypertensiven Blutdruckdysregulationen führte [55]. Wichtig ist jedoch, ausgeprägte hypertensive Phasen zu vermeiden, da diese mit einer erhöhten zerebralen und kardialen Morbidität und Mortalität assoziiert sein können. Die Therapie schwerer hypertensiver Blutdruckentgleisungen sollte intra- und auch postoperativ mit kurz wirksamen Substanzen erfolgen. Hier kommen v. a. Nitroglyzerin und Urapidil infrage. Bei gleichzeitigem Auftreten einer Tachykardie sind kurz wirksame $\beta$ Blocker wie Esmolol eine therapeutische Option. Postoperativ ist die Weiterführung der präoperativen antihypertensiven Therapie empfehlenswert.

Hypotensive Blutdruckwerte gilt es jedoch ebenfalls zu vermeiden, da diese ebenfalls die Inzidenz zerebraler und kardialer Komplikationen erhöhen können. Bei Auftreten hypotensiver Blutdruckdysregulationen ist zunächst die suffiziente Volumentherapie sicherzustellen. Gegebenenfalls ist eine Therapie mit kurz wirksamen vasoaktiven Substanzen erforderlich. Da Blutdruckregulationsstörungen einer der wesentlichsten Trigger 


\begin{tabular}{|c|c|c|c|c|c|c|c|}
\hline \multirow[t]{2}{*}{ Autor } & \multirow[t]{2}{*}{ Jahr } & \multicolumn{6}{|c|}{ Parameter } \\
\hline & & Tod & $\begin{array}{l}\text { Akuter Myo- } \\
\text { kardinfarkt }\end{array}$ & Apoplex & Shunt & Nachblutung & Nervenläsion \\
\hline Binder et al. [10] & 1999 & J & J & J & J & J & J \\
\hline Forssel et al. [32] & 1989 & J & J & J & J & J & $\mathrm{N}$ \\
\hline Kasprzak et al. [40] & 2002 & J & J & J & J & J & J \\
\hline McCarthy et al. [52] & 2001 & J & J & J & $\mathrm{N}$ & $\mathrm{N}$ & $\mathrm{N}$ \\
\hline Pluskwa et al. [68] & 1989 & J & J & J & $\mathrm{N}$ & $\mathrm{N}$ & $\mathrm{N}$ \\
\hline Prough et al. [69] & 1989 & J & J & J & $\mathrm{N}$ & $\mathrm{N}$ & $\mathrm{N}$ \\
\hline Sbarigia et al. [78] & 1999 & J & J & J & J & J & J \\
\hline J ja, N nein. & & & & & & & \\
\hline
\end{tabular}

für postoperative myokardiale Ischämien sind, stellt die konsequente Blutdrucktherapie einen entscheidenden Eckpfeiler in der Vermeidung postoperativer myokardialer Komplikationen dar.

Eine mögliche zerebrale Komplikation ist der postoperative Schlaganfall. Die Gründe hierfür sind vielfältig und umfassen neben einer unzureichenden Kollateralperfusion in der Clampingphase auch schwere hypotensive Blutdruckregulationsstörungen und eine Thrombosierung der ACI oder eine Embolisation im Stromgebiet der ACI, die auch noch postoperativ auftreten kann. Eine besondere neurologische Komplikation ist das Hyperperfusionssyndrom. Hierbei kommt es aufgrund einer gestörten vaskulären Autoregulation im reperfundierten Hirnareal nach Wiederfreigabe der Perfusion zu einer rapiden Steigerung des CBI. In Abhängigkeit vom Ausmaß der Perfusionszunahme und den individuellen zerebralen Kompensationsmöglichkeiten kann die Symptomatik von leichten Kopfschmerzen über das Auftreten von Krampfanfällen bis hin zum Hirnödem oder der intrazerebralen Blutung reichen. Die Wahrscheinlichkeit des Auftretens eines Hyperperfusionssyndroms wird durch mehrere Faktoren wie den Grad der ACI-Stenose oder das Auftreten schwerer hypertensiver Blutdruckentgleisungen bestimmt. Eine mögliche präventive Maßnahme liegt daher in der Vermeidung hypertensiver Blutdruckphasen und der Erhaltung der Normotonie.

Obwohl das Auftreten relevanter Nachblutungen eher die Ausnahme ist, stellen diese jedoch wegen der möglichen Verlegung der Atemwege einen vitalen Notfall dar, der ein sicheres Atemwegsmanagement erfordert.

\section{Vergleich der Anästhesieverfahren}

In den letzten Jahren gab es eine Reihe von Studien, die beide Anästhesieverfahren hinsichtlich der 30-Tage-Mortalität, Schlaganfallhäufigkeit, Shuntfrequenz, Myokardinfarkthäufigkeit, intraoperativen Nervenverletzungen und der Nachblutungshäufigkeit verglichen. Vor dem Hintergrund einer Verschärfung des ökonomischen Drucks auf die Krankenhäuser untersuchten einige Autoren auch die Dauer des Krankenhausaufenthalts in Abhängigkeit vom gewählten Anästhesieverfahren. Die Aussagekraft der Studien wird jedoch dadurch eingeschränkt, dass es sich in der Mehrzahl der Fälle um nichtrandomisierte oder retrospektive Studien handelte.

\section{Randomisierte Untersuchungen}

Im Zeitraum von 1985-2007 wurden 7 das Anästhesieverfahren vergleichende prospektiv randomisierte Studien mit lediglich 554 Operationen durchgeführt [10, 32, 40, 52, 68, 69, 78]. Der Fokus lag hierbei auf der 30-Tage-Mortalität, der Schlaganfallinzidenz und der Myokardinfarkthäufigkeit sowie in der Shuntfrequenz, der Inzidenz an Nervenläsionen und Nachblutungen (• Tab. 6).

\section{0-Tage-Mortalität}

In allen 7 randomisierten Arbeiten war die 30-Tage-Mortalität ein wesentlicher Zielparameter. Hierbei verstarb in den Studien von Binder et al. [10], Prough et al. [69] und Pluskwa et al. [68] weder in der Allgemeinanästhesiegruppe noch in der Regionalanästhesiegruppe ein Patient innerhalb von 30 Tagen. Im Gegen- satz zu diesen Ergebnissen zeigten die Untersuchungen von Forssell et al. [32] Kasprzak et al. [40] und Sbariga et al. [78] eine erhöhte 30-Tage-Mortalität in der Allgemeinanästhesiegruppe, die jedoch keine statistische Signifikanz aufwies. In der Arbeit von McCarthy et al. [52] trat es sowohl in der Regionalanästhesiegruppe als auch in der Allgemeinanästhesiegruppe jeweils ein Todesfall auf. In der Zusammenfassung aller randomisierten Untersuchungen kam es mit 6 Todesfällen in der Allgemeinanästhesiegruppe zu mehr Todesfällen als in der Regionalanästhesiegruppe, in der lediglich ein Fall verzeichnet wurde. Dieser Unterschied kann jedoch nicht als statistisch signifikant bewertet werden.

\section{Myokardinfarktinzidenz}

In den Studien von Binder et al. [10], Kasprzak et al. [40], Pluskwa et al. [68] und Prough et al. [69] zeigte sich kein Unterschied hinsichtlich der Myokardinfarktinzidenz zwischen beiden Anästhesieverfahren, da in keiner dieser Untersuchungen ein Myokardinfarkt verzeichnet werden konnte. Die Arbeiten von Sbarigia et al. [78] und McCarthy et al. [52] zeigten tendenziell weniger Myokardinfarkte in den Regionalanästhesiegruppen. In der Studie von Forssell et al. [32] traten 9 Myokardinfarkte auf: Es ereigneten sich 4 in der Regionalanästhesiegruppe und 5 in der Allgemeinanästhesiegruppe.

\section{Schlaganfallhäufigkeit}

Hinsichtlich der Schlaganfallinzidenz gab es zwischen Allgemeinanästhesie und Regionalanästhesie keine signifikanten Unterschiede. So kam es in den Untersuchungen von Binder et al. [10], McCar- 
thy et al. [52] und Prough et al. [69] weder unter Allgemein- noch unter Regionalanästhesie zu einem Schlaganfall innerhalb von 30 Tagen. In den Studien von Forssell et al. [32] und Kasprzak et al. [40] wurden tendenziell mehr Schlaganfälle in der Regionalanästhesiegruppe beobachtet. Demgegenüber waren in den Untersuchungen von Pluskwa et al. [68] und Sbarigia et al. [78] Schlaganfälle in der Allgemeinanästhesiegruppe häufiger.

\section{Shuntfrequenz}

In lediglich 4 von 7 randomisierten Arbeiten wurde die Shuntfrequenz in Abhängigkeit vom Anästhesieverfahren analysiert. In der Arbeit von Binder et al. [10] erfolgte die routinemäßige Anlage eines endoluminalen Shunts. Somit gab es hier keinen Unterschied zwischen der Allgemeinanästhesie- und der Regionalanästhesiegruppe. Kasprzak et al. [40] berichteten in der Allgemeinanästhesiegruppe über eine niedrigere Shuntfrequenz. Im Gegensatz zeigten die Untersuchungen von Forssell et al. [32] und Sbarigia et al. [78] unter Regionalanästhesie eine geringere Shuntfrequenz. Insgesamt war unter Regionalanästhesie tendenziell eine $\mathrm{Ab}$ nahme der Shuntfrequenz zu verzeichnen. Aufgrund der Heterogenität der Untersuchungen kann jedoch nicht von einer signifikanten Verringerung der Shuntrate gesprochen werden.

\section{Nervenläsionen}

In 3 Untersuchungen wurde der Einfluss des Anästhesieverfahrens auf die Inzidenz von Hirnnervenläsionen evaluiert. Während weder Binder et al. [10] noch Kasprzak et al. [40] einen Einfluss des Anästhesieverfahrens auf die Inzidenz von Hirnnervenläsionen ausmachen konnten, fanden Sbarigia et al. [78] tendenziell mehr Nervenläsionen in der Regionalanästhesiegruppe. Dieser Unterschied war jedoch nicht signifikant.

\section{Nachblutungen}

In den Untersuchungen von Binder et al. [10], Forssell et al. [32], Kasprzak et al. [40] und Sbarigia et al. [78] war der Nachweis der Inzidenz von Nachblutungen eines der Studienziele. Alle 4 Studien belegten eine erhöhte Inzidenz an Nachblutugen unter Allgemeinanästhesie.
Tab. 7 Erfasste Outcomeparameter der nichtrandomisierten Untersuchungen. (Rerkasem et al. [71])

\begin{tabular}{|c|c|c|}
\hline Studienautor & Jahr & Parameter \\
\hline Agrifoglio & 1987 & Tod, AMI, Stroke \\
\hline Allen & 1994 & $\begin{array}{l}\text { Tod, AMI, Stroke, Shunthäufigkeit, Nachblutung, Nervenläsion, pulmo- } \\
\text { nale Komplikationen }\end{array}$ \\
\hline Andersen & 1980 & Tod, Stroke \\
\hline Bartoloni & 1991 & $\begin{array}{l}\text { Tod, AMI, Stroke, Shunthäufigkeit, Nachblutung, Nervenläsion, Pulmo- } \\
\text { nale Komplikationen }\end{array}$ \\
\hline Becquemin & 1991 & Tod, AMI, Stroke, Shunthäufigkeit, Nachblutung, Nervenläsion \\
\hline Bowyer & 2000 & $\begin{array}{l}\text { Tod, AMI, Stroke, Shunthäufigkeit, Nachblutung, Nervenläsion, pulmo- } \\
\text { nale Komplikationen }\end{array}$ \\
\hline Brown & 1999 & Tod, AMl \\
\hline Corson & 1987 & Tod, Stroke, Shunthäufigkeit, Nachblutung, Pulmonale Komplikationen \\
\hline ECST & 1998 & Tod, AMI, Stroke, Shunthäufigkeit, Nachblutung, Nervenläsion \\
\hline Gabelman & 1983 & Tod, AMI, Stroke, Shunthäufigkeit, Nachblutung, Nervenläsion \\
\hline Ghali & 1997 & Tod, Shunthäufigkeit \\
\hline Godin & 1989 & Tod, AMI, Stroke \\
\hline Harbaugh & 2000 & $\begin{array}{l}\text { Tod, AMI, Stroke, Nachblutung, Nervenläsion, Pulmonale Komplika- } \\
\text { tionen }\end{array}$ \\
\hline Hartsell & 1999 & Tod, Stroke, Shunthäufigkeit, Nachblutung, Nervenläsion \\
\hline Imparato & 1998 & Tod, Shunthäufigkeit \\
\hline Jopling & 1983 & Tod, Stroke, Shunthäufigkeit \\
\hline Kraiss & 1995 & Tod, Stroke \\
\hline Kucey & 1998 & Tod \\
\hline Love & 2000 & Tod, Stroke, Shunthäufigkeit, Nachblutung, Nervenläsion \\
\hline McCarthy & 2001 & Tod, AMI, Stroke, Shunthäufigkeit, Nachblutung, Nervenläsion \\
\hline McCleary & 1996 & Tod, Stroke, Shunthäufigkeit \\
\hline Merten & 2000 & Tod \\
\hline Muskett & 1986 & $\begin{array}{l}\text { Tod, AMI, Stroke, Shunthäufigkeit, Nachblutung, Pulmonale Kompli- } \\
\text { kationen }\end{array}$ \\
\hline NASCET & 1998 & Tod, AMI, Stroke, Shunthäufigkeit, Nachblutung, Nervenläsion \\
\hline Ombrellaro & 1996 & Tod, AMI, Stroke, Shunthäufigkeit \\
\hline Palmer & 1989 & Tod, AMI, Stroke, Shunthäufigkeit \\
\hline Quigley & 2000 & Tod, AMI, Stroke, Shunthäufigkeit, Nachblutung, Nervenläsion \\
\hline Reina & 1998 & Tod, Stroke, Shunthäufigkeit \\
\hline Rignano & 1999 & Tod, Shunthäufigkeit \\
\hline Schwartz & 1988 & Tod, AMI, Stroke, Shunthäufigkeit, Pulmonale Komplikationen \\
\hline Shah & 1994 & Tod, AMl, Stroke, Shunthäufigkeit \\
\hline Stone & 2000 & Tod, Stroke, Shunthäufigkeit, Nachblutung, Nervenläsion \\
\hline Stoughton & 1998 & Tod, AMI, Stroke, Shunthäufigkeit \\
\hline Syrek & 1999 & Tod, Stroke \\
\hline Taylor & 1999 & Tod, AMI, Stroke, Shunthäufigkeit \\
\hline Fiorani & 1997 & AMI, Stroke, Shunthäufigkeit \\
\hline Rockman & 1996 & AMI, Stroke \\
\hline Wellman & 1998 & Shunthäufigkeit \\
\hline Karp & 1998 & Stroke und Tod \\
\hline Scuderi & 1998 & Blutdruck \\
\hline Stullken & 1984 & Blutdruck \\
\hline
\end{tabular}

AMI akuter Myokardinfarkt, ECST European Carotid Surgery Trial, NASCET North American Symptomatic Carotid Endarterectomy Trial. 


\section{Pulmonale Komplikationen}

In keiner der 7 randomisierten Untersuchungen wurde über das Auftreten von pulmonalen Komplikationen berichtet.

\section{Hämodynamik}

In 6 der 7 randomisierten Untersuchungen stellte der intra- und postoperative Blutdruckverlauf in Abhängigkeit von der Anästhesietechnik ein Zielkriterium dar. Pluskwa et al. [68], Prough et al. [69], Forssell et al. [32] und McCarthy et al. [52] berichteten über mehr hypotensive Episoden während der Einleitung der Allgemeinanästhesie. Forssell et al. [32] belegten eine signifikante Häufung von intraoperativen hypotensiven Episoden in der Allgemeinanästhesiegruppe. Im postoperativen Verlauf zeigten 2 Autoren [32, 68] allerdings eine Hypotonieneigung nach Regionalanästhesie auf. Im Gegensatz zu McCarthy et al. [52] zeigten Forssell et al. [32], Prough et al. [69] und Pluskwa et al. [68] eine Häufung hypertensiver Episoden während der Clampingphase unter Regionalanästhesie. Postoperativ waren diese hypertensiven Episoden unter Regionalanästhesie aber seltener [40]. Eine vergleichende Beurteilung der Studien war jedoch kaum möglich, da in den einzelnen Arbeiten Hypound Hypertension nicht einheitlich definiert waren. Daher gab es unterschiedliche Interventionsgrenzen in den jeweiligen Untersuchungen.

\section{Krankenhausverweildauer}

Lediglich die Arbeit von Binder et al. [10] untersuchte die Abhängigkeit der Krankenhausverweildauer von der Art des gewählten Anästhesieverfahrens. Während die Verweildauer in der Regionalanästhesiegruppe bei 7,9 Tagen lag, betrug sie in der Allgemeinanästhesiegruppe 9,2 Tage. Dieser Unterschied war jedoch statistisch nicht signifikant.

\section{Nichtradomisierte Untersuchungen}

Im Gegensatz zu der geringen Anzahl an prospektiv randomisierten Untersuchungen existiert eine ungleich größere Menge an retrospektiven Arbeiten. Eine Cochrane-Analyse erfasste 41 nichtrandomisierte Untersuchungen mit insgesamt 25.622 Operationen und verglich das Outcome in Abhängigkeit von der Wahl des Anästhesieverfahrens (• Tab. 7; [71]). Da die Aussagekraft von retrospektiven Arbeiten designbedingt erheblich eingeschränkt ist, können diese allenfalls Hypothesen generierend wirken, nicht aber als evidenzbasierte Grundlage im Vergleich zwischen Regional- und Allgemeinanästhesie dienen.

\section{0-Tage-Mortalität}

In 35 nichtrandomisierten Untersuchungen mit insgesamt 17.702 Operationen - hiervon 8202 in Regional- und 9501 in Allgemeinanästhesie - stellte die Erfassung der 30-Tage-Mortalität ein wesentliches Studienziel dar. Unter Regionalanästhesie war die Mortalität mit 76 Todesfällen im Vergleich zu 116 Todesfällen unter Allgemeinanästhesie signifikant niedriger.

\section{Myokardinfarktinzidenz}

Die Rate an perioperativen Myokardinfarkten war unter Regionalanästhesie mit 84 vs. 239 Ereignissen unter Allgemeinanästhesie ebenfalls signifikant geringer. Dieser Outcomeparameter war in $22 \mathrm{Stu}$ dien mit insgesamt 14.773 Operationen untersucht worden.

\section{Shuntfrequenz}

Eine Aussage hinsichtlich der Shuntfrequenz zu treffen, erscheint anhand der vorliegenden Untersuchungen schwierig. Zwar wurde unter Regionalanästhesie nur bei 13,3\% der Patienten ein Shunt eingelegt, während unter Allgemeinanästhesie 49,5\% der Patienten einen Shunt erhielten. Jedoch wiesen die verschiedenen Untersuchungen einerseits eine starke Heterogenität auf, andererseits existierten keine einheitlichen Kriterien zur Anlage eines endoluminalen Shunts.

\section{Schlaganfallhäufigkeit}

In 31 nichtrandomisierten Untersuchungen wurde über die Inzidenz perioperativer Schlaganfälle berichtet. Unter Allgemeinanästhesie war die Schlaganfallhäufigkeit mit 398 Ereignissen während 8358 Operationen signifikant höher als unter Regionalanästhesie mit 166 Ereignissen bei 8474 Operationen.

\section{Nervenläsionen}

Lediglich in 13 Studien wurde die Inzidenz von Hirnnervenläsionen in Abhängigkeit vom Anästhesieverfahren untersucht. Signifikante Unterschiede konnten nicht aufgezeigt werden.

\section{Nachblutungen}

In 15 Studien wurde die Häufigkeit von Nachblutungen oder Wundhämatomen in Abhängigkeit vom Anästhesieverfahren erfasst. Es zeigten sich ebenfalls keine signifikanten Unterschiede.

\section{Pulmonale Komplikationen}

Lediglich in 7 Untersuchungen war die Inzidenz pulmonaler Komplikationen ein Outcomeparameter. Zwar wurden pulmonale Komplikationen unterschiedlich definiert, traten aber dennoch nach Regionalanästhesie signifikant seltener auf als nach Allgemeinanästhesie.

\section{Hämodynamik}

In 17 nichtrandomisierten Arbeiten wurde der Einfluss des Anästhesieverfahrens auf den perioperativen Blutdruckverlauf untersucht. Intraoperativ waren hypertensive Episoden unter Regionalanästhesie in 4 Untersuchungen häufiger. In einer Untersuchung zeigte sich allerdings unter Allgemeinanästhesie eine höhere Inzidenz hypertensiver Episoden. Postoperativ waren erhöhte Blutdruckwerte meist häufiger nach Allgemeinanästhesie. Allein in einer Untersuchung waren Hypertensionen nach Regionalanästhesie häufiger. Postoperative Hypotensionen waren nach Allgemeinanästhesie ebenfalls häufiger, und nur ein Autor berichtete über eine Häufung postoperativer Hypotensionen nach Regionalanästhesie.

\section{Krankenhausverweildauer}

In 11 Studien war die Erfassung der Krankenhausverweildauer ein Zielparameter, zusätzlich wurde in 4 dieser Arbeiten die Intensivverweildauer verglichen. In allen Untersuchungen zeigte sich sowohl für den Krankenhaus- als auch für den Intensivaufenthalt eine kürzere Verweildauer in der Regionalanästhesiegruppe. Eine erhebliche Einschränkung dieser Aussagen besteht jedoch darin, dass keine klar definierten Entlassungskriterien festgelegt worden waren. 


\section{Fazit für die Praxis}

Trotz des vergleichsweise kleinen chirurgischen Eingriffs weist die TEA der ACl, bedingt durch die temporäre Minderung oder Unterbrechung des CBF und die Komplexität der physiologischen Reaktionen auf den Eingriff per se eine hohe perioperative Morbidität und Mortalität auf. Meist liegt bei diesen Patienten ebenfalls eine signifikante Komorbidität vor.

Alle Faktoren zusammengenommen, stellt das perioperative Management hohe Anforderungen an Operateur und Anästhesist. Das anästhesiologische Vorgehen muss sich daher neben der kontinuierlichen Überwachung der neurologischen Funktion v. a. auf die Sicherstellung einer suffizienten Sauerstoffversorgung von Gehirn und Myokard konzentrieren.

Allgemeinanästhesieverfahren bieten neuroprotektive Effekte und einen höheren Patientenkomfort, erfordern aber ein aufwendiges apparatives neurologisches Monitoring, das auch bei allem Aufwand zerebrale Ischämiephasen nicht absolut zuverlässig detektieren kann. Regionalanästhesieverfahren erleichtern die neurologische Beurteilbarkeit des wachen Patienten, erschweren aber die Führung unkooperativer Patienten. Als zusätzliche Nachteile gelten der ungesicherte Atemweg und die technikbedingten Komplikationsmöglichkeiten selbst. Eine Metaanalyse nichtrandomisierter Untersuchungen zeigte aber Vorteile für die Regionalanästhesietechniken hinsichtlich kardialer Morbidität, Mortalität und kürzerer Krankenhausverweildauer.

Für die Wahl der Regionalanästhesie zur Karotischirurgie anstelle einer Allgemeinanästhesie kann also letztlich aufgrund des Evidenzlevels $1^{-}-2^{+}$allenfalls eine Grad-C-Empfehlung gegeben werden. Um eine endgültige Aussage zum optimalen Anästhesieverfahren zur Karotischirurgie zu treffen, sind weitere ausreichend große prospektiv randomisierte Studien erforderlich.

\section{Korrespondenzadresse Dr. T. Rössel}

Klinik für Anästhesiologie und Intensivmedizin, Universitätsklinikum Dresden Fetscherstraße 74, 01307 Dresden thomas.roessel@uniklinikum-dresden.de

Danksagung. Die Autoren danken PD Dr. med. $\mathrm{H}$ Theilen und Dr. med. J. Schmidt für das zur Verfügung gestellte Bildmaterial und die inhaltliche Diskussion.

\section{Literatur}

1. Executive Committee for the Asymptomatic Carotid Atherosclerosis Study (1995) Endarterectomy for asymptomatic carotid artery stenosis. JAMA 273: $1421-1428$

2. Akbari CM, Pomposelli FB Jr, Gibbons GW et al. (1997) Diabetes mellitus: a risk factor for carotid endarterectomy? J Vasc Surg 25: 1070-1075

3. Al-Rawi PG, Sigaudo-Roussel D, Gaunt ME (2004) Effect of lignocaine injection in carotid sinus on baroreceptor sensitivity during carotid endarterectomy. J Vasc Surg 39: 1288-1294

4. Aleksic M, Gawenda M, Heckenkamp J et al. (2004) Prediction of cerebral ischemic tolerance during carotid cross-clamping by angiographic criteria. Eur J Vasc Endovasc Surg 27: 640-645

5. Alozairi O, Mackenzie RK, Morgan R et al. (2003) Carotid endarterectomy in patients aged 75 and over: early results and late outcome. Eur JVasc Endovasc Surg 26: 245-249

6. Badner NH, Beattie WS, Freeman D, Spence JD (2000) Nitrous oxide-induced increased homocysteine concentrations are associated with increased postoperative myocardial ischemia in patients undergoing carotid endarterectomy. Anesth Analg 91: 1073-1079

7. Baker AB, Roxburgh AJ (1986) Computerised EEG monitoring for carotid endarterectomy. Anaesth Intensive Care 14: 32-36

8. Bamford J, Sandercock P, Dennis M et al. (1990) A prospective study of acute cerebrovascular disease in the community: the Oxfordshire Community Stroke Project 1981-1986. 2. Incidence, case fatality rates and overall outcome at one year of cerebral infarction, primary intracerebral and subarachnoid haemorrhage. J Neurol Neurosurg Psychiatry 53: 16-22

9. Belardi P, Lucertini G, Ermirio D (2003) Stump pressure and transcranial Doppler for predicting shunting in carotid endarterectomy. Eur J Vasc Endovasc Surg 25: 164-167

10. Binder M, Fitzgerald R, Fried H, Schwartz S (1999) Karotisdesobliteration in Cervicalblockade: eine ökonomische Alternative zur Vollnarkose? Gesundheitsoekonom Qualitätsmanage 4: 19-24

11. Bond R, Rerkasem K, Rothwell PM (2003) Routine or selective carotid artery shunting for carotid endarterectomy (and different methods of monitoring in selective shunting). Stroke 34: 824-825

12. Bundesgeschäftsstelle für Qualitätssicherung (2007) BQS-Bundesauswertung 2006. Karotis Rekonstruktion. http://www.bqs-outcome.de/2006/ ergebnisse/leistungsbereiche/karotis/bu_Gesamt 10n2-KARO_2006_9n0.pdf

13. Cao P, Rango P de, Cieri E, Giordano G (2004) Eversion versus conventional endarterectomy. Semin Vasc Surg 17: 236-242

14. Cao P, Giordano G, Zannetti S et al. (1997) Transcranial Doppler monitoring during carotid endarterectomy: is it appropriate for selecting patients in need of a shunt? JVasc Surg 26: 973-979

15. CASANOVA Study Group (1991) Carotid surgery versus medical therapy in asymptomatic carotid stenosis. Stroke 22: 1229-1235

16. Chambers BR, Norris JW (1986) Outcome in patients with asymptomatic neck bruits. N Engl J Med 315 : 860-865

17. Cohen SN, Hobson RW, Weiss DG, Chimowitz M (1993) Death associated with asymptomatic carotid artery stenosis: long-term clinical evaluation. VA Cooperative Study 167 Group. JVasc Surg 18: 1002-1009
18. Collins R, Armitage J, Parish S et al. (2004) Effects of cholesterol-lowering with simvastatin on stroke and other major vascular events in 20,536 people with cerebrovascular disease or other high-risk conditions. Lancet 363: 757-767

19. Davies MJ, Silbert BS, Scott DA et al. (1997) Superficial and deep cervical plexus block for carotid artery surgery: a prospective study of 1,000 blocks. Reg Anesth 22: 442-446

20. Sousa AA de, Dellaretti MA, Faglioni W Jr, Carvalho GT (2005) Monitoring of activated coagulation time in carotid endarterectomy. Surg Neurol 64 [Suppl 1] S1-S9

21. Sousa AA de, Filho MA, Faglione $W \mathrm{Jr}$, Carvalho GT (2005) Superficial vs combined cervical plexus block for carotid endarterectomy: a prospective, randomized study. Surg Neurol 63 [Suppl 1]: S22-S25

22. Castro V de, Godet G, Mencia G et al. (2003) Targetcontrolled infusion for remifentanil in vascular patients improves hemodynamics and decreases remifentanil requirement. Anesth Analg 96:33-38; table

23. Dinkel M, Langer $H$, Loerler H et al. (1994) Neuromonitoring in carotid surgery: possibilities and limits of transcranial Doppler ultrasound. Vasa 23: 337-344

24. Doyle PW, Coles JP, Leary TM et al. (2001) A comparison of remifentanil and fentanyl in patients undergoing carotid endarterectomy. Eur J Anaesthesiol 18: 13-19

25. Eckstein $\mathrm{HH}$ (2004) [Surgical therapy of extracranial carotid stenosis]. Chirurg 75: 93-110

26. Eckstein HH, Maeder N, Allenberg JR (1999) [Carotid surgery for prophylaxis of ischemic stroke]. Chirurg 70:353-363

27. European Carotid Surgery Trialists Collaborative Group (1995) Risk of stroke in the distribution of an asymptomatic carotid artery. Lancet 345: 209-212

28. European Carotid Surgery Trialists Collaborative Group (1998) Randomised trial of endarterectomy for recently symptomatic carotid stenosis: final results of the MRC European Carotid Surgery Trial (ECST). Lancet 351: 1379-1387

29. Fava E, Bortolani E, Ducati A, Schieppati M (1992) Role of SEP in identifying patients requiring temporary shunt during carotid endarterectomy. Electroencephalogr Clin Neurophysiol 84: 426-432

30. Ferguson GG, Eliasziw M, Barr HW et al. (1999) The North American Symptomatic Carotid Endarterec tomy Trial: surgical results in 1415 patients. Stroke 30: 1751-1758

31. Fisher M, Davalos A (2004) The MATCH study results in the context of secondary stroke prevention. Stroke 35: 2609

32. Forssell C, Takolander R, Bergqvist D et al. (1989) Local versus general anaesthesia in carotid surgery. A prospective, randomised study. Eur J Vasc Surg 3: 503-509

33. Ghali R, Palazzo EG, Rodriguez DI et al. (1997) Transcranial Doppler intraoperative monitoring during carotid endarterectomy: experience with regional or general anesthesia, with and without shunting. Ann Vasc Surg 11: 9-13

34. Godet G, Watremez C, El Kettani Cet al. (2001) A comparison of sevoflurane, target-controlled infusion propofol, and propofol/isoflurane anesthesia in patients undergoing carotid surgery: a quality of anesthesia and recovery profile. Anesth Analg 93: $560-565$

35. Graham DI (1985) The pathology of brain ischaemia and possibilities for therapeutic intervention. $\mathrm{Br} \mathrm{J}$ Anaesth 57: 3-17

36. Halliday A, Mansfield A, Marro J et al. (2004) Prevention of disabling and fatal strokes by successfu carotid endarterectomy in patients without recent neurological symptoms: randomised controlled trial. Lancet 363: 1491-1502 
37. Hans SS, Jareunpoon O (2007) Prospective evaluation of electroencephalography, carotid artery stump pressure, and neurologic changes during 314 consecutive carotid endarterectomies performed in awake patients. J Vasc Surg 45: 511-515

38. Hertzer NR, Flanagan RA Jr, Beven EG, O'Hara PJ (1986) Surgical versus nonoperative treatment of asymptomatic carotid stenosis. 290 patients documented by intravenous angiography. Ann Surg 204: 163-171

39. Jellish WS, Sheikh T, Baker WH et al. (2003) Hemodynamic stability, myocardial ischemia, and perioperative outcome after carotid surgery with remifentanil/propofol or isoflurane/fentanyl anesthesia. J Neurosurg Anesthesiol 15: 176-184

40. Kasprzak PM, Altmeppen J, Angerer M et al. (2006) General versus locoregional anesthesia in carotid surgery: a prospective randomised trial. Vasa 35 : 232-238

41. Kastrup A, Schulz JB, Raygrotzki S et al. (2004) Comparison of angioplasty and stenting with cerebral protection versus endarterectomy for treatment of internal carotid artery stenosis in elderly patients. J Vasc Surg 40: 945-951

42. Kim GE, Cho YP, Lim SM (2002) The anatomy of the circle of Willis as a predictive factor for intra-operative cerebral ischemia (shunt need) during carotid endarterectomy. Neurol Res 24: 237-240

43. Kim JS, Moon DH, Kim GE et al. (2000) Acetazolamide stress brain-perfusion SPECT predicts the need for carotid shunting during carotid endarterectomy. J Nucl Med 41: 1836-1841

44. Kozmary SV, Lampe GH, Benefiel D et al. (1990) No finding of increased myocardial ischemia during or after carotid endarterectomy under anesthesia with nitrous oxide. Anesth Analg 71:591-596

45. Kwaan JH, Peterson GJ, Connolly JE (1980) Stump pressure: an unreliable guide for shunting during carotid endarterectomy. Arch Surg 115: 1083-1086

46. Lacroix H, Beyens G, Hemelrijck J van et al. (1999) Is transcranial Doppler useful in the detection of internal carotid artery cross-clamp intolerance? Cardiovasc Surg 7: 203-207

47. Lam AM, Manninen PH, Ferguson GG, Nantau W (1991) Monitoring electrophysiologic function during carotid endarterectomy: a comparison of somatosensory evoked potentials and conventional electroencephalogram. Anesthesiology 75: 15-21

48. Lang W, Dinkel M (2000) Cerebral ischemia during carotid clamping: diagnosis and prevention. Zentralbl Chir 125: 243-250

49. Marietta DR, Lunn JK, Ruby El, Hill GE (1998) Cardiovascular stability during carotid endarterectomy: endotracheal intubation versus laryngeal mask airway. J Clin Anesth 10: 54-57

50. Mattos MA, Sumner DS, Bohannon WT et al. (2001) Carotid endarterectomy in women: challenging the results from ACAS and NASCET. Ann Surg 234: 438445

51. Mayberg MR, Wilson SE, Yatsu F et al. (1991) Carotid endarterectomy and prevention of cerebral is chemia in symptomatic carotid stenosis. Veterans Affairs Cooperative Studies Program 309 Trialist Group. JAMA 266: 3289-3294

52. McCarthy RJ, Nasr MK, McAteer P, Horrocks M (2002) Physiological advantages of cerebral blood flow during carotid endarterectomy under local anaesthesia. A randomised clinical trial. Eur J Vasc Endovasc Surg 24: 215-221

53. McCulloch TJ, Thompson CL, Turner MJ (2007) A randomized crossover comparison of the effects of propofol and sevoflurane on cerebral hemodynamics during carotid endarterectomy. Anesthesiology 106: 56-64

54. McFarland HR, Pinkerton JA Jr, Frye D (1988) Continuous electroencephalographic monitoring during carotid endarterectomy. J Cardiovasc Surg (Torino) 29: $12-18$
55. Mehta M, Rahmani O, Dietzek AM et al. (2001) Eversion technique increases the risk for post-carotid endarterectomy hypertension. JVasc Surg 34: 839-845

56. Mehta Y, Juneja R (1992) Regional analgesia for carotid artery endarterectomy by Winnie's singleinjection technique using a nerve detector 135 . J Cardiothorac Vasc Anesth 6: 772-773

57. Merle JC, Mazoit JX, Desgranges P et al. (1999) A comparison of two techniques for cervical plexus blockade: evaluation of efficacy and systemic toxicity. Anesth Analg 89: 1366-1370

58. Messick JM Jr, Casement B, Sharbrough FW et al. (1987) Correlation of regional cerebral blood flow ( $\mathrm{CBF}$ ) with EEG changes during isoflurane anesthesia for carotid endarterectomy: critical rCBF. Anesthesiology 66: 344-349

59. Michel A, Weigand MA, Eckstein HH et al. (2000) Measurement of local oxygen parameters for detection of cerebral ischemia. The significance of cerebral near-infrared spectroscopy and transconjunctival oxygen partial pressure in carotid surgery. Anaesthesist 49: 392-401

60. Moen V, Dahlgren N, Irestedt L (2004) Severe neurological complications after central neuraxial blockades in Sweden 1990-1999. Anesthesiology 101: 950-959

61. Moore DC (1975) Regional block: a handbook for use in the clinical practice of medicine and surgery, 4 th edn. Thomas, Springfield, pp 112-122

62. Mouren S, Winter G de, Guerrero SP et al. (2001) The continuous recording of blood pressure in patients undergoing carotid surgery under remifentanil versus sufentanil analgesia. Anesth Analg 93: 14021409; table

63. North American Symptomatic Carotid Endarterectomy Trial Collaborators (1991) Beneficial effect of carotid endarterectomy in symptomatic patients with high-grade carotid stenosis. N Engl J Med 325: 445-453

64. Paciaroni M, Eliasziw M, Kappelle L et al. (1999) Medical complications associated with carotid endarterectomy. North American Symptomatic Carotid Endarterectomy Trial (NASCET). Stroke 30: 17591763

65. Pandit JJ, Bree S, Dillon P et al. (2000) A comparison of superficial versus combined (superficial and deep) cervical plexus block for carotid endarterectomy: a prospective, randomized study. Anesth Analg 91: 781-786

66. Pandit JJ, Dutta D, Morris JF (2003) Spread of injectate with superficial cervical plexus block in humans: an anatomical study. Br J Anaesth 91: 733-735

67. Pandit JJ, Satya-Krishna R, Gration P (2007) Superficial or deep cervical plexus block for carotid endarterectomy: a systematic review of complications. Br J Anaesth 99: 159-169

68. Pluskwa F, Bonnet F, Abhay K et al. (1989) Comparison of blood pressure profiles with flunitrazepam/ fentanyl/nitrous oxide vs cervical epidural anesthesia in surgery of the carotid artery. Ann Fr Anesth Reanim 8: 26-32

69. Prough DS, Scuderi PE, McWhorter JM et al. (1989) Hemodynamic status following regional and general anesthesia for carotid endarterectomy. J Neurosurg Anesthesiol 1: 35-40

70. Rampil IJ, Holzer JA, Quest DO et al. (1983) Prognostic value of computerized EEG analysis during carotid endarterectomy. Anesth Analg 62: 186-192

71. Rerkasem K, Bond R, Rothwell PM (2004) Local versus general anaesthesia for carotid endarterectomy. Cochrane Database Syst Rev 2: CD000126

72. Rihal CS, Gersh BJ, Whisnant JP et al. (1992) Influence of coronary heart disease on morbidity and mortality after carotid endarterectomy: a population-based study in Olmsted County, Minnesota (1970-1988). J Am Coll Cardiol 19: 1254-1260
73. Ringleb PA, Allenberg J, Bruckmann $\mathrm{H}$ et al. (2006) 30 day results from the SPACE trial of stent-protected angioplasty versus carotid endarterectomy in symptomatic patients: a randomised non-inferiority trial. Lancet 368: 1239-1247

74. Roessel T, Wiessner D, Heller AR et al. (2007) Highresolution ultrasound-guided high interscalene plexus block for carotid endarterectomy. Reg Anesth Pain Med 32: 247-253

75. Russ W, Fraedrich G, Hehrlein FW, Hempelmann G (1985) Intraoperative somatosensory evoked potentials as a prognostic factor of neurologic state after carotid endarterectomy. Thorac Cardiovasc Surg 33: 392-396

76. Sacco RL, Foulkes MA, Mohr JP et al. (1989) Determinants of early recurrence of cerebral infarction. The Stroke Data Bank. Stroke 20: 983-989

77. Sandmann W, Kolvenbach R, Willeke F (1993) Risks and benefits of shunting in carotid endarterectomy. Stroke 24: 1098-1099

78. Sbarigia E, DarioVizza C, Antonini M et al. (1999) Locoregional versus general anesthesia in carotid surgery: is there an impact on perioperative myocardial ischemia? Results of a prospective monocentric randomized trial. J Vasc Surg 30: 131-138

79. Sbarigia E, Schioppa A, Misuraca M et al. (2001) Somatosensory evoked potentials versus locoregional anaesthesia in the monitoring of cerebral function during carotid artery surgery: preliminary results of a prospective study. Eur JVasc Endovasc Surg 21:413-416

80. Schneemilch CE, Ludwig S, Ulrich A et al. (2007) Somatosensory evoked potentials and biochemical markers of neuronal deficits in patients undergoing carotid endarterectomy under regional anesthesia. Zentralbl Chir 132: 176-182

81. Seraglio P, Campostella FA (2001) Cervical plexus block and continuous cervical peridural block with ropivacaine for carotid surgery: a comparison between the 2 methods. Minerva Anestesiol 67: $65-70$

82. Stoneham MD, Bree SE (1999) Epileptic seizure during awake carotid endarterectomy. Anesth Analg 89: 885-886

83. Stoneham MD, Doyle AR, Knighton JD et al. (1998) Prospective, randomized comparison of deep or superficial cervical plexus block for carotid endarterectomy surgery. Anesthesiology 89: 907-912

84. Taylor DW, Barnett HJ, Haynes RB et al. (1999) Lowdose and high-dose acetylsalicylic acid for patients undergoing carotid endarterectomy: a randomised controlled trial. ASA and Carotid Endarterectomy (ACE) Trial Collaborators. Lancet 353: 2179-2184

85. Tempelhoff R, Modica PA, Grubb RL Jr et al. (1989) Selective shunting during carotid endarterectomy based on two-channel computerized electroencephalographic/compressed spectral array analysis. Neurosurgery 24: 339-344

86. Umbrain V, Keeris J, D'Haese J et al. (2000) Isoflurane, desflurane and sevoflurane for carotid endarterectomy. Anaesthesia 55: 1052-1057

87. Vollmar J (1996) Rekonstruktive Chirurgie der Arterien, 4. Aufl. Thieme, Stuttgart, S 392-395

88. Weimar C, Diener HC (2004) Diagnose und Therapie der Schlaganfallbehandlung in Deutschland. Dtsch Arztebl 100: 2576-2582

89. Wilhelm W, Schlaich N, Harrer J et al. (2001) Recovery and neurological examination after remifentanildesflurane or fentanyl-desflurane anaesthesia for carotid artery surgery. Br J Anaesth 86: 44-49

90. Winnie AP, Ramamurthy S, Durrani Z, Radonjic R (1975) Interscalene cervical plexus block: a singleinjection technic. Anesth Analg 54: 370-375

91. Yadav JS, Wholey MH, Kuntz RE et al. (2004) Protected carotid-artery stenting versus endarterectomy in high-risk patients. N Engl J Med 351: 14931501 